\title{
Whitney towers, gropes and Casson-Gordon style invariants of links
}

\author{
Min HoOn KiM
}

\begin{abstract}
In this paper, we prove a conjecture of Friedl and Powell that their Casson-Gordon type invariant of 2-component links with linking number one is actually an obstruction to being height-3.5 Whitney tower/grope concordant to the Hopf link. The proof employs the notion of solvable cobordism of 3-manifolds with boundary, which was introduced by Cha. We also prove that the Blanchfield form and the Alexander polynomial of links in $S^{3}$ give obstructions to height- 3 Whitney tower/grope concordance. This generalizes the results of Hillman and Kawauchi.
\end{abstract}

57M25, 57M27; 57N70

\section{Introduction}

In the study of topological knot concordance, various invariants were introduced in seminal papers including Levine [32], Casson and Gordon [3; 4], and Cochran, Orr and Teichner [15]. All of these invariants can be extracted from the 0 -surgery manifolds of knots. Influenced by these works, the link slicing problem has been studied extensively using various covers of the 0-surgery manifolds of links. For example, Harvey [26], Cochran, Harvey and Leidy [11] and Horn [29] used Cheeger-Gromov $\rho$-invariants from PTFA (poly-torsion-free-abelian) covers. In Cha [5; 6], Hirzebruch type invariants from iterated prime power fold covers were defined and used.

In general link concordance problems, it is known that 0 -surgery manifolds do not reveal full information. For example, for 2-component links with linking number one, the aforementioned invariants automatically vanish. In fact, those invariants are obtained from solvable covers of 0 -surgery manifolds. For 2-component links with linking number one, there are no nontrivial solvable covers of the 0 -surgery manifolds (and consequently the aforementioned invariants vanish) because they have perfect fundamental groups. Also, there is an in-depth study which presents related results about link concordance versus 0-surgery homology cobordism; see Cha and Powell [9].

Recently, for 2-component links with linking number one, S Friedl and M Powell [25] introduced a Casson-Gordon style metabelian invariant $\tau(L, \chi)$ by considering another 
closed 3-manifold obtained from the link exterior. Also, they found new 2-component links with linking number one which are not concordant to the Hopf link. The aim of this paper is to give a better understanding of $\tau(L, \chi)$ in the context of symmetric Whitney towers and gropes in dimension 4.

\section{Friedl-Powell invariant $\tau(L, \chi)$}

To describe our main result, we briefly summarize the construction and main result in [25]. (For more details, see Section 4.)

Let $L$ be an ordered, oriented 2-component link with linking number 1 in $S^{3}$ and $H$ be the Hopf link. Define $M_{L}$ to be the closed 3-manifold obtained by gluing the exteriors of $L$ and $H$ along their boundary, identifying the meridians of corresponding components. For a prime $p$, choose a homomorphism $\varphi: H_{1}\left(M_{L} ; \mathbb{Z}\right) \rightarrow \mathbb{Z} / p^{i} \times \mathbb{Z} / p^{j}$ which sends two meridians of $L$ to the standard basis $(1,0)$ and $(0,1)$, respectively. Let $M_{L}^{\varphi} \rightarrow M_{L}$ be the $p^{i+j}$-fold covering induced by $\varphi$. For a prime $q$ and a character $\chi: H_{1}\left(M_{L}^{\varphi} ; \mathbb{Z}\right) \rightarrow \mathbb{Z} / q^{k}$, Friedl and Powell define an invariant

$$
\tau(L, \chi) \in L^{0}(\mathbb{C}(\mathcal{H})) \otimes_{\mathbb{Z}} \mathbb{Z}[1 / q]
$$

in [25, Section 3.2] (see also our Definition 4.1). Here, $\mathcal{H}=\mathbb{Z}^{3}, \mathbb{C}(\mathcal{H})$ is the quotient field of the group ring $\mathbb{C}[\mathcal{H}]$, and $L^{0}(\mathbb{C}(\mathcal{H}))$ is the Witt group of finite-dimensional nonsingular sesquilinear forms over $\mathbb{C}(\mathcal{H})$. The main result of [25] essentially says that if $L$ is concordant to $H$, then $\tau(L, \chi)$ vanishes [25, Theorem 3.5]. For a precise definition of the vanishing of $\tau(L, \chi)$, see Definition 4.2. We omit the precise statement here because we would need to discuss some technicalities including the choice of a metabolizer of the linking form.

\section{Symmetric Whitney tower/grope concordance and $\tau(L, \chi)$}

The symmetric Whitney towers and gropes are approximations of embedded surfaces which play a central role in the study of topological 4-manifolds. For example, a special kind of grope with caps gives a topologically embedded disk in the disk embedding theorem of Freedman and Quinn [23]. Also, using symmetric Whitney towers and gropes, $\mathrm{T}$ Cochran, $\mathrm{K}$ Orr and $\mathrm{P}$ Teichner developed a filtration theory of the knot concordance group [15]. It turns out that the structure of this filtration theory is extremely rich (for example, see Cochran, Orr and Teichner [16], Cochran and Teichner [17], Cochran, Harvey and Leidy [12; 13], Cha [7], Davis [21], Franklin [22] and Burke [2]). For links, we are mainly interested in two equivalence relations, height $h$ Whitney tower concordance and height $h$ grope concordance. (For precise definitions, see Cha [8, Definitions 2.12, 2.15].) 
We remark that J Conant, R Schneiderman, and Teichner developed another interesting filtration theory using a coarser notion called order- $n$ Whitney tower concordance (for a survey and references, we refer to [18]). It is not our purpose to study this asymmetric filtration theory of Conant, Schneiderman and Teichner. We focus on the finer equivalence relations, symmetric Whitney tower/grope concordance.

Our main result, Theorem A, says that the Friedl-Powell invariant $\tau(L, \chi)$ can be understood in terms of symmetric Whitney tower/grope concordance as conjectured in [25, Remark 1.3(5)]:

Theorem A Suppose that $L$ is a 2-component link with linking number 1 and $H$ is the Hopf link. If $L$ and $H$ are height-3.5 Whitney tower (or grope) concordant, then the Friedl-Powell invariant $\tau(L, \chi)$ vanishes for $L$ in the sense of Definition 4.2.

In the proof, we use the notion of $h$-solvable cobordism, introduced by $\mathrm{J} C$ Cha in [8] (for the definition, see Section 3.1). By [8, Theorem 2.13], if two links $L$ and $L^{\prime}$ are height- $(h+2)$ Whitney tower/grope concordant, then their exteriors $X_{L}$ and $X_{L^{\prime}}$ are $h$-solvable cobordant for all $h \in \frac{1}{2} \mathbb{Z}_{\geq 0}$. Actually, we prove Theorem A in Section 4.3 under the (potentially) weaker assumption that there exists a 1.5-solvable cobordism between the exteriors $X_{L}$ and $X_{H}$.

Remark (1) In [15, Theorem 9.11], Cochran, Orr and Teichner proved that if a knot $K$ bounds a Whitney tower/grope of height 3.5 in $D^{4}$, or more generally if $K$ is $1.5-$ solvable, then the Casson-Gordon invariant $\tau(K, \chi)$ vanishes. Our result can be viewed as an analogue for 2-component links with linking number 1 .

(2) Theorem A is strictly stronger than [25, Theorem 3.5] by the following fact from [8, Theorem 4.1]: for any integer $n>2$, there are links which are height- $n$ grope concordant to $H$ but not height- $n .5$ Whitney tower concordant to $H$ (in particular, not concordant to $H$ ).

\section{Symmetric Whitney tower/grope concordance and abelian invariants}

In [15, Theorem 1.1], Cochran, Orr and Teichner proved that a Seifert form of a knot $K$ is metabolic if and only if $K$ bounds a height- 2.5 grope in $D^{4}$. By Schneiderman [35, Corollary 2] and [15, Theorem 8.12], this condition is equivalent to that $K$ bounds a height-2.5 Whitney tower in $D^{4}$. Motivated from this result, in Section 5 we prove that Blanchfield form and the multivariable Alexander polynomial are actually obstructions to height-3 Whitney tower/grope concordance.

Abelian link concordance invariants are studied by A Kawauchi [30] and J Hillman [28]. To state our main result, we recall their notation (for details, see Section 5) and main 
results. Let $L$ be a $\mu$-component link and let $X_{L}$ be the exterior of $L$. Denote $\mathbb{Z}\left[t_{1}^{ \pm}, \ldots, t_{\mu}^{ \pm}\right]$by $\Lambda_{\mu}$. The ring $\Lambda_{\mu}$ is endowed with the involution $-: t_{i} \mapsto t_{i}^{-1}$. Let $S$ be the multiplicative set generated by $\left\{t_{1}-1, \ldots, t_{\mu}-1\right\}$. Denote by $\Lambda_{\mu} S$ the localization of $\Lambda_{\mu}$ with respect to $S$. Let $\mathcal{K}$ be the quotient field of $\Lambda_{\mu}$. Using the Hurewicz map $\pi_{1}\left(X_{L}\right) \rightarrow \mathbb{Z}^{\mu}$, we define $H_{*}\left(X_{L} ; \Lambda_{\mu}\right)$ and $H_{*}\left(X_{L} ; \Lambda_{\mu S}\right)$.

In [28, Chapter 2], Hillman defined the $\mathcal{K} / \Lambda_{\mu S}$-valued localized Blanchfield form $b_{L}$ on the quotient of the torsion submodule of $H_{1}\left(X_{L} ; \Lambda_{\mu S}\right)$ by its maximal pseudonull submodule. Also, he proved that the Witt-class of $b_{L}$, denoted by $\left[b_{L}\right]$, in the Witt group $W\left(\mathcal{K}, \Lambda_{\mu S},-\right)$ is a link concordance invariant.

In [30], Kawauchi defined the torsion Alexander polynomial of $L$ which we denote by $\Delta_{L}^{T}$. In [30, Theorems A, B], he proved that if two links $L_{0}$ and $L_{1}$ are concordant, then $\operatorname{rank}_{\Lambda_{\mu}} H_{1}\left(X_{L_{0}} ; \Lambda_{\mu}\right)=\operatorname{rank}_{\Lambda_{\mu}} H_{1}\left(X_{L_{1}} ; \Lambda_{\mu}\right)$ and $\Delta_{L_{0}}^{T} f_{0} \overline{f_{0}} \doteq \Delta_{L_{1}}^{T} f_{1} \overline{f_{1}}$ for some $f_{i}\left(t_{1}, \ldots, t_{\mu}\right) \in \Lambda_{\mu}, i=0,1$ with $\left|f_{i}(1, \ldots, 1)\right|=1$.

We extend these theorems of Hillman and Kawauchi in terms of symmetric Whitney tower/grope concordance as follows:

Theorem B Suppose that two links $L_{0}$ and $L_{1}$ are height-3 Whitney tower/grope concordant. Then $\left[b_{L_{0}}\right]=\left[b_{L_{1}}\right] \in W\left(\mathcal{K}, \Lambda_{\mu S},-\right)$.

Theorem C Suppose that two links $L_{0}$ and $L_{1}$ are height-3 Whitney tower/grope concordant. Then:

(1) $\operatorname{rank}_{\Lambda_{\mu}} H_{1}\left(X_{L_{0}} ; \Lambda_{\mu}\right)=\operatorname{rank}_{\Lambda_{\mu}} H_{1}\left(X_{L_{1}} ; \Lambda_{\mu}\right)$.

(2) $\Delta_{L_{0}}^{T} f_{0} \overline{f_{0}} \doteq \Delta_{L_{1}}^{T} f_{1} \bar{f}_{1}$ for some $f_{i}\left(t_{1}, \ldots, t_{\mu}\right) \in \Lambda_{\mu}, i=0,1$ with

$$
\left|f_{i}(1, \ldots, 1)\right|=1 \text {. }
$$

As a special case of Theorems B and C for 2-component links with linking number 1, we have the following special case. This illustrates that the concordance problem between a 2-component link with linking number 1 and the Hopf link is similar to the concordance problem between a knot and the unknot.

Corollary D Suppose that $L$ is a 2-component link with linking number 1 and $H$ is the Hopf link. If $L$ and $H$ are height-3 Whitney tower/grope concordant, then:

(1) $\left[b_{L}\right]=0 \in W\left(\mathcal{K}, \Lambda_{2},-\right)$.

(2) $\operatorname{rank}_{\Lambda_{2}} H_{1}\left(X_{L} ; \Lambda_{2}\right)=0$.

(3) $\Delta_{L}^{T} \doteq f \bar{f}$ for some $f\left(t_{1}, t_{2}\right) \in \Lambda_{2}$ such that $|f(1,1)|=1$. 
Remark Theorems B and C should be compared to the following equivalent statements for knots about abelian invariants (eg [15, Theorem 1.1] and Kearton [31]).

(1) The knot $K$ bounds a grope of height 2.5 in $D^{4}$.

(2) The 0 -surgery manifold of $K, M_{K}$ is 0.5 -solvable.

(3) The Seifert form of $K$ is metabolic (or $K$ is algebraically slice).

(4) The Blanchfield form of $K$ is Witt-trivial.

Therefore, the most natural assumption for Theorems B and C might be the existence of 0.5-solvable cobordism between link exteriors. The proof for the knot case heavily relies on the existence of Seifert surfaces for $K$. For general links, as substitutes for Seifert surfaces, there are immersed Cooper surfaces studied in Cooper [19] (or its generalization in Cimasoni [10]). However, because of their singularities, a similar approach using Cooper surface seems somewhat difficult.

Acknowledgements The author would like to express his deep gratitude to his advisor Jae Choon Cha for suggesting the problem and for many valuable conversations about this work. He also would like to thank Stefan Friedl, Jonathan Hillman and Mark Powell for their helpful suggestions. This research was supported by a National Research Foundation of Korea Grant funded by the Korean Government (NRF-2011-0002353).

\section{Casson-Gordon type representations}

The goal of this section is to prove Theorem 2.3 which will give the key dimension estimate in the proof of Theorem A. Lemma 2.2 and Theorem 2.3 are inspired by [7, Lemma 3.10 and Theorem 3.11]. In the proof of Lemma 2.2, we use the injectivity theorem of Friedl and Powell [24, Theorem 3.1] stated in Lemma 2.1.

We recall the notation used in [24] for the convenience of the reader. Let $\varphi: G \rightarrow A$ be a surjective group homomorphism, where $A$ is a finite abelian $p$-group. Assume that $\varphi: G \rightarrow A$ factors through a surjective homomorphism $\phi^{\prime}: G \rightarrow \mathcal{H}^{\prime}$ to a torsion free abelian group $\mathcal{H}^{\prime}$. Let $K=\operatorname{Ker} \varphi, \mathcal{H}=\operatorname{Im}\left(\left.\phi^{\prime}\right|_{K}\right)$ and $\phi: K \rightarrow \mathcal{H}$ be the restriction of $\phi^{\prime}$ to $K$. Note that $\mathcal{H}$ is a torsion free abelian group. In short, we have the following commutative diagram:

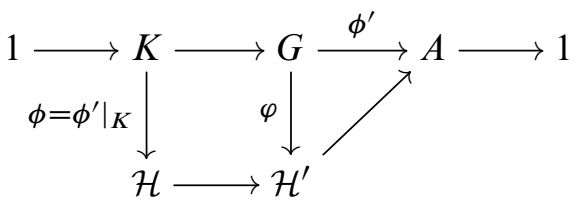


Suppose that $\alpha: K \rightarrow \operatorname{GL}(d, Q)$ is a $d$-dimensional representation to a field $Q$ of characteristic zero such that $\left.\alpha\right|_{\operatorname{Ker} \phi}$ factors through a $q$-group for some prime $q$. Let $Q(\mathcal{H})$ be the quotient field of the group ring $Q[\mathcal{H}]$. Note that $\alpha$ and $\phi$ give a right $\mathbb{Z} K$-module structure on $Q^{d} \otimes_{Q} Q(\mathcal{H})=Q(\mathcal{H})^{d}$ as follows:

$$
\begin{aligned}
Q^{d} \otimes_{Q} Q(\mathcal{H}) \times \mathbb{Z} K & \rightarrow Q^{d} \otimes_{Q} Q(\mathcal{H}), \\
(v \otimes p, g) & \mapsto(v \cdot \alpha(g) \otimes p, \phi(g)) .
\end{aligned}
$$

We write $t=|A| . \mathbb{Z} G$ is a left (rank $t$ free) $\mathbb{Z} K$-module. Note that there is a right action of $G$ on $d t$-dimensional $Q$-vector space $Q^{d} \otimes_{\mathbb{Z} K} \mathbb{Z} G$. Equivalently, there is an induced representation $\alpha^{\prime}: G \rightarrow \mathrm{GL}(d t, Q)$.

As in (2-1), $\alpha^{\prime}$ and $\phi^{\prime}$ give a right $\mathbb{Z} G$-module structure on $Q\left(\mathcal{H}^{\prime}\right)^{d t}=Q^{d t} \otimes_{Q} Q\left(\mathcal{H}^{\prime}\right)$ :

$$
\begin{aligned}
Q^{d t} \otimes_{Q} Q\left(\mathcal{H}^{\prime}\right) \times \mathbb{Z} G & \rightarrow Q^{d t} \otimes_{Q} Q\left(\mathcal{H}^{\prime}\right), \\
(v \otimes p, g) & \mapsto\left(v \cdot \alpha^{\prime}(g) \otimes p, \phi^{\prime}(g)\right) .
\end{aligned}
$$

$\operatorname{Regard} \mathbb{Z} / q$ as a $\mathbb{Z} G$-module with the trivial $G$-action.

Lemma 2.1 [24, Theorem 3.1] Let $f: M \rightarrow N$ be a morphism of projective left $\mathbb{Z} G$-modules such that

$$
1_{\mathbb{Z} / q} \otimes_{\mathbb{Z} G} f: \mathbb{Z} / q \otimes_{\mathbb{Z} G} M \rightarrow \mathbb{Z} / q \otimes_{\mathbb{Z} G} N
$$

is injective. Then

$$
1_{Q\left(\mathcal{H}^{\prime}\right)}{ }^{d t} \otimes_{\mathbb{Z} G} f: Q\left(\mathcal{H}^{\prime}\right)^{d t} \otimes_{\mathbb{Z} G} M \rightarrow Q\left(\mathcal{H}^{\prime}\right)^{d t} \otimes_{\mathbb{Z} G} N
$$

is injective.

Using Lemma 2.1, we prove Lemma 2.2 and Theorem 2.3.

Lemma 2.2 Let $f: M \rightarrow N$ be a morphism of left $\mathbb{Z} G$-modules.

(1) If $N$ is projective, then

$$
d t \cdot \operatorname{dim}_{\mathbb{Z} / q} \operatorname{Im}\left(1_{\mathbb{Z} / q} \otimes_{\mathbb{Z} G} f\right) \leq \operatorname{dim}_{Q\left(\mathcal{H}^{\prime}\right)} \operatorname{Im}\left(1_{Q\left(\mathcal{H}^{\prime}\right)} d t \otimes_{\mathbb{Z} G} f\right) .
$$

(2) If, in addition, $M$ is finitely generated and free, then

$$
\left.d t \cdot \operatorname{dim}_{\mathbb{Z} / q} \operatorname{Ker}\left(1_{\mathbb{Z} / q} \otimes_{\mathbb{Z} G} f\right) \geq \operatorname{dim}_{Q\left(\mathcal{H}^{\prime}\right)} \operatorname{Ker}\left(1_{Q\left(\mathcal{H}^{\prime}\right)}\right)^{d t} \otimes_{\mathbb{Z} G} f\right) .
$$


Proof (1) Let $k=\operatorname{dim}_{\mathbb{Z} / q} \operatorname{Im}\left(1_{\mathbb{Z} / q} \otimes_{\mathbb{Z} G} f\right)$ ( $k$ may be any cardinal number.) Note that $\mathbb{Z} / q \otimes_{\mathbb{Z} G}-$ induces two surjections $(\mathbb{Z} G)^{k} \rightarrow(\mathbb{Z} / q)^{k}$ and $\operatorname{Im} f \rightarrow$ $\operatorname{Im}\left(1_{\mathbb{Z} / q} \otimes_{\mathbb{Z} G} f\right)$. Since $(\mathbb{Z} G)^{k}$ is free, there exists a homomorphism $i:(\mathbb{Z} G)^{k} \rightarrow$ $\operatorname{Im} f$ such that the following diagram commutes:

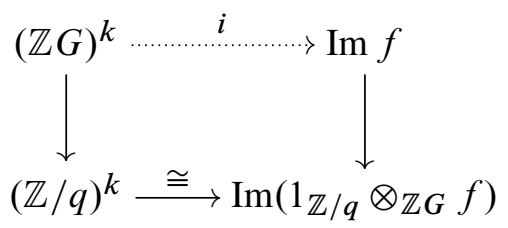

Recall that $N$ is a projective $\mathbb{Z} G$-module. Obviously, $(\mathbb{Z} G)^{k}$ is a projective $\mathbb{Z} G-$ module. Hence, we can apply Lemma 2.1 to $i:(\mathbb{Z} G)^{k} \rightarrow \operatorname{Im} f \subset N$ and obtain the injection

$$
1_{Q\left(\mathcal{H}^{\prime}\right)}^{d t} \otimes_{\mathbb{Z} G} i: Q\left(\mathcal{H}^{\prime}\right)^{d t k}=Q\left(\mathcal{H}^{\prime}\right)^{d t} \otimes_{\mathbb{Z} G}(\mathbb{Z} G)^{k} \hookrightarrow Q\left(\mathcal{H}^{\prime}\right)^{d t} \otimes_{\mathbb{Z} G} N .
$$

Since $\operatorname{Im} i \subset \operatorname{Im} f$,

$$
Q\left(\mathcal{H}^{\prime}\right)^{d t k} \cong \operatorname{Im}\left(1_{Q\left(\mathcal{H}^{\prime}\right)}^{d t} \otimes_{\mathbb{Z} G} i\right) \subset \operatorname{Im}\left(1_{Q\left(\mathcal{H}^{\prime}\right)}{ }^{d t} \otimes_{\mathbb{Z} G} f\right) .
$$

This implies

$$
d t \cdot \operatorname{dim}_{\mathbb{Z} / q} \operatorname{Im}\left(1_{\mathbb{Z} / q} \otimes_{\mathbb{Z} G} f\right)=d t k \leq \operatorname{dim}_{Q\left(\mathcal{H}^{\prime}\right)}\left(1_{Q\left(\mathcal{H}^{\prime}\right)} d t \otimes_{\mathbb{Z} G} f\right) .
$$

(2) Let $M=(\mathbb{Z} G)^{n}$. Part (1) and the following elementary observation complete the proof:

$\operatorname{dim}_{\mathbb{Z} / q} \operatorname{Ker}\left(1_{\mathbb{Z} / q} \otimes_{\mathbb{Z} G} f\right)+\operatorname{dim}_{\mathbb{Z} / q} \operatorname{Im}\left(1_{\mathbb{Z} / q} \otimes_{\mathbb{Z} G} f\right)=\operatorname{dim}_{\mathbb{Z} / q} \mathbb{Z} / q \otimes_{\mathbb{Z} G} M=n$.

Similarly,

$$
\left.n d t=\operatorname{dim}_{Q\left(\mathcal{H}^{\prime}\right)} \operatorname{Ker}\left(1_{Q\left(\mathcal{H}^{\prime}\right)}\right)^{d t} \otimes_{\mathbb{Z} G} f\right)+\operatorname{dim}_{Q\left(\mathcal{H}^{\prime}\right)} \operatorname{Im}\left(1_{Q\left(\mathcal{H}^{\prime}\right)} d t \otimes_{\mathbb{Z} G} f\right) .
$$

Theorem 2.3 Suppose $C_{*}$ is a chain complex of projective left $\mathbb{Z} G$-modules with $C_{n}$ finitely generated. Let $\left\{x_{i}\right\}_{i \in I}$ be a collection of $n$-cycles in $C_{n}$. Let $M$ be the $\left(Q(\mathcal{H})^{d} \otimes_{\mathbb{Z} K} \mathbb{Z} G\right)$-span of $\left\{\left[1_{Q(\mathcal{H})^{d}} \otimes_{\mathbb{Z} K} x_{i}\right]_{i \in I}\right.$. Let $\bar{M}$ be the $\mathbb{Z} / q$-span of $\left\{\left[1_{\mathbb{Z} / q} \otimes_{\mathbb{Z} G} x_{i}\right]\right\}_{i \in I}$. Then we have

$$
\operatorname{dim}_{Q(\mathcal{H})} H_{n}\left(Q(\mathcal{H})^{d} \otimes_{\mathbb{Z} K} C_{*}\right) / M \leq d t \cdot \operatorname{dim}_{\mathbb{Z} / q} H_{n}\left(\mathbb{Z} / q \otimes_{\mathbb{Z} G} C_{*}\right) / \bar{M} .
$$

Proof Let $\partial_{n}: C_{n} \rightarrow C_{n-1}$ be the boundary map of $C_{*}$ and define $f:(\mathbb{Z} G)^{I} \oplus$ $C_{n+1} \rightarrow C_{n}$ by $\left(e_{i}, v\right) \mapsto x_{i}+\partial_{n+1}(v)$, where $\left\{e_{i}\right\}_{i \in I}$ is the standard basis of $(\mathbb{Z} G)^{I}$. 
Then we have

$$
\begin{gathered}
H_{n}\left(Q(\mathcal{H})^{d} \otimes_{\mathbb{Z} K} C_{*}\right) / M=\operatorname{Ker}\left(1_{Q(\mathcal{H})^{d}} \otimes_{\mathbb{Z} K} \partial_{n}\right) / \operatorname{Im}\left(1_{Q(\mathcal{H})^{d}} \otimes_{\mathbb{Z} K} f\right), \\
H_{n}\left(\mathbb{Z} / q \otimes_{\mathbb{Z} G} C_{*}\right) / \bar{M}=\operatorname{Ker}\left(1_{\mathbb{Z} / q} \otimes_{\mathbb{Z} G} \partial_{n}\right) / \operatorname{Im}\left(1_{\mathbb{Z} / q} \otimes_{\mathbb{Z} G} f\right) .
\end{gathered}
$$

From the $\mathbb{Z} G$-module structure on $Q\left(\mathcal{H}^{\prime}\right)^{d t}$ in (2-2),

$$
\begin{aligned}
Q\left(\mathcal{H}^{\prime}\right) \otimes_{Q(\mathcal{H})} Q(\mathcal{H})^{d} \otimes_{\mathbb{Z} K} \mathbb{Z} G & =\left(Q\left(\mathcal{H}^{\prime}\right) \otimes_{Q(\mathcal{H})} Q(\mathcal{H})^{d} \otimes_{\mathbb{Z} K} \mathbb{Z} G\right) \otimes_{\mathbb{Z} G} \mathbb{Z} G \\
& =Q\left(\mathcal{H}^{\prime}\right)^{d t} \otimes_{\mathbb{Z} G} \mathbb{Z} G .
\end{aligned}
$$

Since $C_{*}$ is a chain complex of left $\mathbb{Z} G$-modules,

$$
Q\left(\mathcal{H}^{\prime}\right) \otimes_{Q(\mathcal{H})} Q(\mathcal{H})^{d} \otimes_{\mathbb{Z} K} C_{*}=Q\left(\mathcal{H}^{\prime}\right)^{d t} \otimes_{\mathbb{Z} G} C_{*} .
$$

Since $\mathcal{H} \hookrightarrow \mathcal{H}^{\prime}, Q\left(\mathcal{H}^{\prime}\right)$ is a flat right $Q(\mathcal{H})$-module. Therefore, we have

$$
H_{*}\left(Q\left(\mathcal{H}^{\prime}\right)^{d t} \otimes_{\mathbb{Z} G} C_{*}\right)=Q\left(\mathcal{H}^{\prime}\right) \otimes_{Q(\mathcal{H})} H_{*}\left(Q(\mathcal{H})^{d} \otimes_{\mathbb{Z} K} C_{*}\right) .
$$

Note that the dimension is left unchanged by $Q\left(\mathcal{H}^{\prime}\right) \otimes Q(\mathcal{H})$. From (2-4), it follows that

$$
\begin{aligned}
\operatorname{dim}_{Q(\mathcal{H})} H_{n}\left(Q(\mathcal{H})^{d} \otimes_{\mathbb{Z} K}\right. & \left.C_{*}\right) / M \\
& =\operatorname{dim}_{Q\left(\mathcal{H}^{\prime}\right)} Q\left(\mathcal{H}^{\prime}\right) \otimes_{Q(\mathcal{H})}\left(H_{n}\left(Q(\mathcal{H})^{d} \otimes_{\mathbb{Z} K} C_{*}\right) / M\right) \\
& =\operatorname{dim}_{Q\left(\mathcal{H}^{\prime}\right)} H_{n}\left(Q\left(\mathcal{H}^{\prime}\right)^{d t} \otimes_{\mathbb{Z} G} C_{*}\right) /\left(Q\left(\mathcal{H}^{\prime}\right) \otimes_{Q(\mathcal{H})} M\right) .
\end{aligned}
$$

Similarly to (2-3),

$$
\begin{aligned}
& H_{n}\left(Q\left(\mathcal{H}^{\prime}\right)^{d t} \otimes_{\mathbb{Z} G} C_{*}\right) /\left(Q\left(\mathcal{H}^{\prime}\right) \otimes_{Q(\mathcal{H})} M\right) \\
& \left.\quad=\operatorname{Ker}\left(1_{Q\left(\mathcal{H}^{\prime}\right)}\right)^{d t} \otimes_{\mathbb{Z} G} \partial_{n}\right) / \operatorname{Im}\left(1_{Q\left(\mathcal{H}^{\prime}\right)^{d t}} \otimes_{\mathbb{Z} G} f\right) .
\end{aligned}
$$

From the above observations and the inequality from Lemma 2.2,

$$
\begin{aligned}
\operatorname{dim}_{Q(\mathcal{H})} H_{n}\left(Q(\mathcal{H})^{d} \otimes_{\mathbb{Z} K} C_{*}\right) / M \\
\quad=\operatorname{dim}_{Q\left(\mathcal{H}^{\prime}\right)} H_{n}\left(Q\left(\mathcal{H}^{\prime}\right)^{d t} \otimes_{\mathbb{Z} G} C_{*}\right) /\left(Q\left(\mathcal{H}^{\prime}\right) \otimes_{Q(\mathcal{H})} M\right) \\
\left.\quad=\operatorname{dim}_{Q\left(\mathcal{H}^{\prime}\right)} \operatorname{Ker}\left(1_{Q\left(\mathcal{H}^{\prime}\right) d t} \otimes_{\mathbb{Z} G} \partial_{n}\right)-\operatorname{dim}_{Q\left(\mathcal{H}^{\prime}\right)} \operatorname{Im}\left(1_{Q\left(\mathcal{H}^{\prime}\right)}\right)_{t} \otimes_{\mathbb{Z} G} f\right) \\
\leq d t\left(\operatorname{dim}_{\mathbb{Z} / q} \operatorname{Ker}\left(1_{\mathbb{Z} / q} \otimes_{\mathbb{Z} G} \partial_{n}\right)-\operatorname{dim}_{\mathbb{Z} / q} \operatorname{Im}\left(1_{\mathbb{Z} / q} \otimes_{\mathbb{Z} G} f\right)\right) \\
\quad=d t \cdot \operatorname{dim}_{\mathbb{Z} / q} H_{n}\left(\mathbb{Z} / q \otimes_{\mathbb{Z} G} C_{*}\right) / \bar{M} .
\end{aligned}
$$

This completes the proof.

We remark that in this paper we use Theorem 2.3 only for a finite collection $I$. 


\section{An $h$-solvable cobordism}

In this section, we give the definition of an $h$-solvable cobordism following [8]. Also, we prove Proposition 3.2 about prime power coverings of 1-solvable cobordisms.

\subsection{Definition of $\boldsymbol{h}$-solvable cobordism}

For oriented compact bordered 3-manifolds $M$ and $M^{\prime}$, a cobordism $W$ between $M$ and $M^{\prime}$ is a 4-dimensional manifold with boundary $\partial W=M \cup_{\partial}-M^{\prime}$, where $-M^{\prime}$ denotes $M^{\prime}$ with reversed orientation. We often denote a cobordism by $\left(W ; M, M^{\prime}\right)$. A cobordism $\left(W ; M, M^{\prime}\right)$ is an $H_{1}$-cobordism (respectively, a homology cobordism) if $H_{i}(M ; \mathbb{Z}) \cong H_{i}(W ; \mathbb{Z}) \cong H_{i}\left(M^{\prime} ; \mathbb{Z}\right)$ under the inclusion map for $i \leq 1$ (respectively, for all $i)$. Note that $H_{2}(W, M)$ is a free abelian group if $\left(W ; M, M^{\prime}\right)$ is an $H_{1}-$ cobordism (for example, see [7, Lemma 3.7]). A cobordism $\left(W ; M, M^{\prime}\right)$ is an $H_{1}-$ cobordism with $\mathbb{Q}$-coefficients if $H_{i}(M ; \mathbb{Q}) \cong H_{i}(W ; \mathbb{Q}) \cong H_{i}\left(M^{\prime} ; \mathbb{Q}\right)$ under the inclusion map for $i \leq 1$.

Example If $L$ is a link in $S^{3}$, then the link exterior $X_{L}$ is a bordered 3-manifold with a canonical homeomorphism between a disjoint union of tori and $\partial X_{L}$ sending the standard basis to the meridians and 0 -framed longitudes of $L$. If two links $L$ and $L^{\prime}$ are concordant, then $X_{L}$ and $X_{L^{\prime}}$ are homology cobordant bordered 3-manifolds via a concordance exterior and $h$-solvable cobordant for all $h \in \frac{1}{2} \mathbb{Z}_{\geq 0}$ (see the definition of solvable cobordism given below).

We use the following notation for covering maps associated to the derived series.

Convention (1) For a space $X$, there is a sequence of regular covers over $X$

$$
X^{(n+1)} \rightarrow X^{(n)} \rightarrow \cdots \rightarrow X^{(1)} \rightarrow X^{(0)}=X
$$

which corresponds to the derived series

$$
\pi^{(n+1)} \subset \pi^{(n)} \subset \cdots \subset \pi^{(0)}=\pi, \quad \text { where } \pi=\pi_{1}(X) \text { and } \pi^{(n+1)}=\left[\pi^{(n)}, \pi^{(n)}\right] \text {. }
$$

With this, we can always identify $H_{*}\left(X ; \mathbb{Z}\left[\pi / \pi^{(n)}\right]\right)=H_{*}\left(X^{(n)} ; \mathbb{Z}\right)$ as usual.

(2) For a 4-manifold $W$ with $\pi=\pi_{1}(W)$, let

$$
\lambda_{n}: H_{2}\left(W ; \mathbb{Z}\left[\pi / \pi^{(n)}\right]\right) \times H_{2}\left(W ; \mathbb{Z}\left[\pi / \pi^{(n)}\right]\right) \rightarrow \mathbb{Z}\left[\pi / \pi^{(n)}\right]
$$

be the intersection form.

(3) For a covering map $Y \rightarrow X$, $\operatorname{Cov}(Y \mid X)$ denotes its deck transformation group.

The action of $\operatorname{Cov}(Y \mid X)$ on $H_{*}(Y ; \mathbb{Z})$ is a right action. 
Definition 3.1 Suppose $\left(W ; M, M^{\prime}\right)$ is an $H_{1}$-cobordism between bordered 3manifolds $M$ and $M^{\prime}$ with $\pi=\pi_{1}(W)$. Let $r=\frac{1}{2} \operatorname{rank} H_{2}(W, M ; \mathbb{Z})$.

(1) A submodule $L \subset H_{2}\left(W ; \mathbb{Z}\left[\pi / \pi^{(n)}\right]\right)$ is an $n$-Lagrangian if $L$ projects to a half-rank summand of $H_{2}(W, M ; \mathbb{Z})$ and $\lambda_{n}$ vanishes on $L$.

(2) For an $n$-Lagrangian $L(k \leq n)$, homology classes

$$
d_{1}, \ldots, d_{r} \in H_{2}\left(W ; \mathbb{Z}\left[\pi / \pi^{(k)}\right]\right)
$$

are $k$-duals if $L$ is generated by $l_{1}, \ldots, l_{r} \in L$ whose projections $l_{1}^{\prime}, \ldots, l_{r}^{\prime} \in$ $H_{2}\left(W ; \mathbb{Z}\left[\pi / \pi^{(k)}\right]\right)$ satisfy $\lambda_{k}\left(l_{i}^{\prime}, d_{j}\right)=\delta_{i j}$.

(3) An $H_{1}$-cobordism $\left(W ; M, M^{\prime}\right)$ is called an $n .5$-solvable cobordism (respectively, $n$-solvable cobordism) if it has an $(n+1)$-Lagrangian (respectively, $n$-Lagrangian) admitting $n$-duals. If there exists an $h$-solvable cobordism from $M$ to $M^{\prime}$, we say that $M$ is $h$-solvable cobordant to $M^{\prime}$ for $h \in \frac{1}{2} \mathbb{Z}_{\geq 0}$.

\subsection{Prime power cover of 1-solvable cobordism}

In this subsection, we prove Proposition 3.2 about the (abelian) prime power cover of 1-solvable cobordism for later purpose. For a finitely generated abelian group $G$, denote the torsion subgroup of $G$ by $t G$ and the free part of $G$ by $F G=G / t G$.

Proposition 3.2 Suppose $\left(W ; M, M^{\prime}\right)$ is a 1 -solvable cobordism with $\varphi: \pi_{1}(W) \rightarrow A$ be a surjective group homomorphism to an abelian $p$-group $A$ and $p$ is prime. We denote the cobordism of the induced coverings by $\left(W^{\varphi} ; M^{\varphi}, M^{\prime \varphi}\right)$. Then:

(1) $\beta_{2}\left(W^{\varphi}, M^{\varphi}\right)=|A| \beta_{2}(W, M)$ where $\beta_{2}$ is the second Betti number.

(2) The inclusion induced map $\mathrm{FH}_{2}\left(W^{\varphi} ; \mathbb{Z}\right) \rightarrow F H_{2}\left(W^{\varphi}, M^{\varphi} ; \mathbb{Z}\right)$ is surjective.

(3) $\left(W^{\varphi} ; M^{\varphi}, M^{\prime \varphi}\right)$ is an $H_{1}$-cobordism with $\mathbb{Q}$-coefficients.

Proof (1) Fix a (relative) CW-complex structure on $(W, M)$. This induces a (relative) $\mathrm{CW}$-complex structure on $\left(W^{\varphi}, M^{\varphi}\right)$. Let $C_{*}=C_{*}\left(W^{\varphi}, M^{\varphi} ; \mathbb{Z}\right)$. Then $C_{*}$ is a chain complex of free $\mathbb{Z} A$-modules and $C_{*}(W, M ; \mathbb{Z})=C_{*} \otimes_{\mathbb{Z} A} \mathbb{Z}$. Since $\left(W ; M, M^{\prime}\right)$ is an $H_{1}$-cobordism, $H_{i}\left(C_{*} \otimes_{\mathbb{Z} A} \mathbb{Z} / p\right)=0$ for $i=0,1$ by the universal coefficient theorem. Since $p$ is prime, Levine's well-known chain homotopy lifting argument [34] shows that $H_{i}\left(C_{*} \otimes_{\mathbb{Z}} \mathbb{Z} / p\right)=0$ for $i=0,1$. In particular, by the universal coefficient theorem, $H_{i}\left(C_{*}\right)$ is a torsion abelian group for $i=0,1$. By the universal coefficient theorem, $H_{i}\left(W^{\varphi}, M^{\varphi} ; \mathbb{Q}\right)=H_{i}\left(C_{*}\right) \otimes_{\mathbb{Z}} \mathbb{Q}=0$ for $i=0,1$. 
By taking $C_{*}=C_{*}\left(W^{\varphi}, M^{\prime \varphi} ; \mathbb{Z}\right)$, the same argument shows $H_{i}\left(W^{\varphi}, M^{\prime \varphi} ; \mathbb{Q}\right)=0$ for $i=0,1$. By Poincaré duality and the universal coefficient theorem,

$$
H_{i}\left(W^{\varphi}, M^{\varphi} ; \mathbb{Q}\right) \cong \operatorname{Hom}_{\mathbb{Q}}\left(H_{4-i}\left(W^{\varphi}, M^{\prime \varphi} ; \mathbb{Q}\right), \mathbb{Q}\right)=0 \quad \text { for } i=3,4 .
$$

So, $\beta_{2}\left(W^{\varphi}, M^{\varphi}\right)=\chi\left(W^{\varphi}, M^{\varphi}\right)$ where $\chi$ is the Euler characteristic. Similarly, $\chi(W, M)=\beta_{2}(W, M)$ because $H_{i}(W, M ; \mathbb{Z})=H_{i}\left(W, M^{\prime} ; \mathbb{Z}\right)=0$ for $i=0,1$. By definition, $\left(W^{\varphi}, M^{\varphi}\right)$ is an $A$-cover of $(W, M)$ and $\chi\left(W^{\varphi}, M^{\varphi}\right)=|A| \chi(W, M)$. This completes the proof of (1).

(2) Since $W^{\varphi} \rightarrow W$ is an abelian covering with $\operatorname{Cov}\left(W^{\varphi} \mid W\right)=A, \pi_{1}(W)^{(1)} \subset$ $\pi_{1}\left(W^{\varphi}\right)$. The covering map $W^{(1)} \rightarrow W^{\varphi}$ induces $H_{2}\left(W^{(1)} ; \mathbb{Z}\right) \rightarrow H_{2}\left(W^{\varphi} ; \mathbb{Z}\right)$. Let $l_{1}, \ldots, l_{r}, d_{1}, \ldots, d_{r}$ be the images of the (generators of) a 1 -Lagrangian and 1 -duals in $H_{2}\left(W^{\varphi} ; \mathbb{Z}\right)$. By the definition of 1 -solvable cobordism, $\beta_{2}(W, M)=2 r$. Let $A=\left\{g_{1}, \ldots, g_{t}\right\}$. From (1), $\beta_{2}\left(W^{\varphi}, M^{\varphi}\right)=\beta_{2}(W, M)|A|=2 r t$.

From the (right) group action of $\mathrm{A}$ on $\mathrm{H}_{2}\left(W^{\varphi} ; \mathbb{Z}\right)$, we can define

$$
l_{i j}=l_{i} \cdot g_{j} \quad \text { and } \quad d_{k l}=d_{k} \cdot g_{l} \quad \text { for } 1 \leq i, k \leq r \text { and } 1 \leq j, l \leq t .
$$

By the definition of a 1-Lagrangian and of 1-duals, the intersection pairing

$$
\lambda: F H_{2}\left(W^{\varphi}, M^{\varphi} ; \mathbb{Z}\right) \times F H_{2}\left(W^{\varphi}, M^{\prime \varphi} ; \mathbb{Z}\right) \rightarrow \mathbb{Z}
$$

on the image of $\left\{l_{i j}, d_{k l}\right\}$ is given by

$$
\left(\begin{array}{cc}
0 & I_{r t \times r t} \\
I_{r t \times r t} & X
\end{array}\right)
$$

Since this matrix has determinant 1 and $\beta_{2}\left(W^{\varphi}, M^{\varphi}\right)=2 r t$, the image of $\left\{l_{i j}, d_{k l}\right\}$ is a basis of $F_{2}\left(W^{\varphi}, M^{\varphi} ; \mathbb{Z}\right)$. This proves inc $*: F H_{2}\left(W^{\varphi} ; \mathbb{Z}\right) \rightarrow F H_{2}\left(W^{\varphi}, M^{\varphi} ; \mathbb{Z}\right)$ is surjective because $\left\{l_{i j}, d_{k l}\right\} \subset F H_{2}\left(W^{\varphi} ; \mathbb{Z}\right)$.

(3) From (2), inc $*: H_{2}\left(W^{\varphi} ; \mathbb{Q}\right) \rightarrow H_{2}\left(W^{\varphi}, M^{\varphi} ; \mathbb{Q}\right)$ is surjective. From (1) and the homology long exact sequence of a pair $\left(W^{\varphi}, M^{\varphi}\right)$, inc $*: H_{i}\left(M^{\varphi} ; \mathbb{Q}\right) \rightarrow H_{i}\left(W^{\varphi} ; \mathbb{Q}\right)$ is an isomorphism for $i=0,1$. The same argument works for $\left(W, M^{\prime}\right)$. This completes the proof.

\section{Solvable cobordism and Friedl-Powell invariant}

Throughout this section, for any finitely generated abelian group $G, t G$ and $F G$ denote the torsion part of $G$ and the free part of $G$, respectively. $G^{\wedge}$ denotes $\operatorname{Hom}_{\mathbb{Z}}(G, \mathbb{Q} / \mathbb{Z})$. For a finite abelian group $G, G^{\wedge}=\operatorname{Ext}_{\mathbb{Z}}(G, \mathbb{Z})$ since $\operatorname{Hom}_{\mathbb{Z}}(G, \mathbb{Q})=\operatorname{Ext}_{\mathbb{Z}}(G, \mathbb{Q})=0$. $H_{*}(-)$ denotes homology with integral coefficients. 


\subsection{Definition of the Friedl-Powell invariant $\tau(L, \chi)$}

To define the Friedl-Powell invariant $\tau(L, \chi)$, we set up the notation and conventions used in [25]. Here, $L$ is a 2-component link with linking number 1 and $H$ is the Hopf link. We denote the exterior of $J$ by $X_{J}=S^{3}-v(J)$ for $J=H, L$. We can decompose $\partial X_{L}$ into $Y_{a} \cup Y_{b}$ with $Y_{a} \cong Y_{b}=S^{1} \times D^{1} \sqcup S^{1} \times D^{1}$ where both $Y_{a}$ and $Y_{b}$ are annuli neighborhood of (parallels of) meridians of $L$. Define $M_{J}=X_{J} \cup_{\partial X_{H} \times I}-X_{H}$ for $J=H, L$ where the gluing map respects the ordering of the link components and identifies each of the subsets $Y_{a}, Y_{b} \subset \partial X_{J}$ for $J=L, H$.

For a prime $p$, we say a group homomorphism $\varphi: H_{1}\left(M_{L}\right) \rightarrow \mathbb{Z} / p^{i} \oplus \mathbb{Z} / p^{j}$ is admissible if $\varphi$ sends the two meridians of $L$ to the standard basis $(1,0),(0,1)$. (From the Mayer-Vietoris sequence, $H_{1}\left(M_{L}\right) \cong H_{1}\left(X_{L}\right) \oplus \mathbb{Z} \cong \mathbb{Z}^{3}$.) Let $M_{L}^{\varphi} \rightarrow M_{L}$ be the $p^{i+j}$-fold covering space from $\varphi$. We denote the Hurewicz map by $\phi^{\prime}: \pi_{1}\left(M_{L}\right) \rightarrow$ $H_{1}\left(M_{L}\right)$. Define $\phi: \pi_{1}\left(M_{L}^{\varphi}\right) \rightarrow H_{1}\left(M_{L}\right)$ to be the restriction $\left.\phi^{\prime}\right|_{\pi_{1}\left(M_{L}^{\varphi}\right)}$ and $\mathcal{H}=$ $\operatorname{Im} \phi$. Choose an isomorphism $\psi: \pi_{1}\left(T^{3}\right) \cong \mathcal{H}$. (Note that $\mathcal{H}$ is isomorphic to $\mathbb{Z}^{3}$ as a finite-index subgroup of $H_{1}\left(M_{L}\right) \cong \mathbb{Z}^{3}$.)

For a prime power character $\chi: \pi_{1}\left(M_{L}^{\varphi}\right) \rightarrow \mathbb{Z} / q^{k}$, we have the bordism class

$$
\left[\left(M_{L}^{\varphi}, \chi \times \phi\right) \sqcup-\left(T^{3}, \operatorname{tr} \times \psi\right)\right] \in \Omega_{3}\left(\mathbb{Z} / q^{k} \times \mathcal{H}\right),
$$

where tr: $\pi_{1}\left(T^{3}\right) \rightarrow \mathbb{Z} / q^{k}$ is the trivial group homomorphism. From the AtiyahHirzebruch spectral sequence calculation given in [25, Section 3.2], [ $\left(M_{L}^{\varphi}, \chi \times \phi\right) \sqcup$ $\left.-\left(T^{3}, \operatorname{tr} \times \psi\right)\right]$ is $q$-primary torsion in $\Omega_{3}\left(\mathbb{Z} / q^{k} \times \mathcal{H}\right)$. In other words, there exist a nonnegative integer $s$, a cobordism $W$ between $q^{s} M_{L}^{\varphi}$ and $q^{s} T^{3}$, and $\Phi: \pi_{1}(W) \rightarrow$ $\mathbb{Z} / q^{k} \times \mathcal{H}$ such that the following diagram commutes:

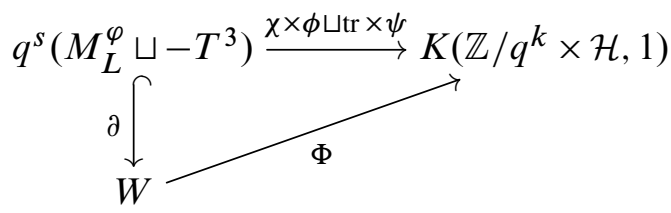

From the sequence of ring homomorphisms

$$
\mathbb{Z}\left[\pi_{1}(W)\right] \stackrel{\Phi}{\rightarrow} \mathbb{Z}\left[\mathbb{Z} / q^{k} \times \mathcal{H}\right]=\mathbb{Z}\left[\mathbb{Z} / q^{k}\right][\mathcal{H}] \rightarrow \mathbb{Q}\left(\xi_{q^{k}}\right)(\mathcal{H}) \rightarrow \mathbb{C}(\mathcal{H})=\mathcal{K},
$$

we can define the twisted intersection form $H_{2}(W ; \mathcal{K}) \times H_{2}(W ; \mathcal{K}) \rightarrow \mathcal{K}$. We denote the nonsingular part of the intersection form on $H_{2}(W ; \mathcal{K})$ (respectively, on $H_{2}(W ; \mathbb{Q})$ ) by $\lambda_{\mathcal{K}}(W)$ (respectively, $\lambda_{\mathbb{Q}}(W)$ ). 
Definition 4.1 (Friedl-Powell invariant) We have

$$
\tau(L, \chi)=\left(\lambda_{\mathcal{K}}(W)-\mathcal{K} \otimes \lambda_{\mathbb{Q}}(W)\right) \otimes \frac{1}{q^{s}} \in L^{0}(\mathcal{K}) \otimes_{\mathbb{Z}} \mathbb{Z}[1 / q],
$$

where $L^{0}(\mathcal{K})$ is the Witt group of finite-dimensional nonsingular sesquilinear forms over $\mathcal{K}$.

In [25, Section 3.2], it is shown that $\tau(L, \chi)$ is well defined. That is, $\tau(L, \chi)$ depends neither on the choice of $W$ nor on the choice of isomorphism $\psi: \pi_{1}\left(T^{3}\right) \rightarrow \mathcal{H}$.

In Section 4.2, we will describe the linking form

$$
\lambda_{L}: t H_{1}\left(X_{L}^{\varphi}, Y_{a}^{\varphi}\right) \times t H_{1}\left(X_{L}^{\varphi}, Y_{a}^{\varphi}\right) \rightarrow \mathbb{Q} / \mathbb{Z}
$$

Now we give the precise statement that the Friedl-Powell invariant vanishes.

Definition 4.2 For 2-component link $L$ with linking number 1, we say the FriedlPowell invariant vanishes for $L$ if for any admissible homomorphism $\varphi: H_{1}\left(M_{L}\right) \rightarrow$ $\mathbb{Z} / p^{i} \otimes \mathbb{Z} / p^{j}$ and for a prime $p$, there exists a metabolizer $P=P^{\perp}$ of the linking form

$$
\lambda_{L}: t H_{1}\left(X_{L}^{\varphi}, Y_{a}^{\varphi}\right) \times t H_{1}\left(X_{L}^{\varphi}, Y_{a}^{\varphi}\right) \rightarrow \mathbb{Q} / \mathbb{Z}
$$

with the following property: For any character of prime-power order $\chi: H_{1}\left(M_{L}^{\varphi}\right) \rightarrow$ $\mathbb{Z} / q^{k}$ which satisfies that $\left.\chi\right|_{H_{1}\left(X_{L}^{\varphi}\right)}$ factors through

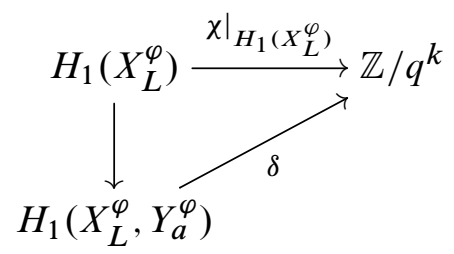

and that $\delta$ vanishes on $P, \tau(L, \chi)=0 \in L^{0}(\mathcal{K}) \otimes_{\mathbb{Z}} \mathbb{Z}[1 / q]$.

The following main theorem will be proved in Section 4.3:

Theorem A Suppose that $L$ is a 2-component link with linking number 1 and $H$ is the Hopf link. If $X_{L}$ and $X_{H}$ are 1.5-solvable cobordant, then the Friedl-Powell invariant $\tau(L, \chi)$ vanishes for $L$ in the sense of Definition 4.2. In particular, the conclusion holds if $L$ and $H$ are height-3.5 Whitney tower/grope concordant. 


\subsection{1-solvable cobordism and a metabolizer of the linking form}

In this subsection, we recall the definition of the linking form

$$
\lambda_{L}: t H_{1}\left(X_{L}^{\varphi}, Y_{a}^{\varphi}\right) \times t H_{1}\left(X_{L}^{\varphi}, Y_{a}^{\varphi}\right) \rightarrow \mathbb{Q} / \mathbb{Z}
$$

defined in [25] and prove Proposition 4.3. The adjoint of $\lambda_{L}, \operatorname{Ad}\left(\lambda_{L}\right): t H_{1}\left(X_{L}^{\varphi}, Y_{a}^{\varphi}\right) \rightarrow$ $t H_{1}\left(X_{L}^{\varphi}, Y_{a}^{\varphi}\right)^{\wedge}$, can be obtained by composing the following isomorphisms:

(a) $t H_{1}\left(X_{L}^{\varphi}, Y_{a}^{\varphi}\right) \rightarrow t H^{2}\left(X_{L}^{\varphi}, Y_{b}^{\varphi}\right) \rightarrow \operatorname{Ext}_{\mathbb{Z}}\left(t H_{1}\left(X_{L}^{\varphi}, Y_{b}^{\varphi}\right), \mathbb{Z}\right)=t H_{1}\left(X_{L}^{\varphi}, Y_{a}^{\varphi}\right)^{\wedge}$.

To see this, we use Poincaré duality, the universal coefficient theorem, $H_{1}\left(X_{L}^{\varphi}, Y_{b}^{\varphi}\right) \cong$ $H_{1}\left(X_{L}^{\varphi}, Y_{a}^{\varphi}\right)$ and that $t H_{1}\left(X_{L}^{\varphi}, Y_{a}^{\varphi}\right)$ is a finite abelian group.

Let $\left(W_{0} ; X_{L}, X_{H}\right)$ be a 1 -solvable cobordism. Recall $\varphi: H_{1}\left(M_{L}\right) \rightarrow \mathbb{Z} / p^{i} \oplus \mathbb{Z} / p^{j}$ is an admissible homomorphism and $H_{1}\left(M_{L}\right) \cong H_{1}\left(X_{L}\right) \oplus \mathbb{Z}$. Then, $\left.\varphi\right|_{H_{1}\left(X_{L}\right)}$ extends to $H_{1}\left(W_{0}\right)$ canonically because $H_{1}\left(X_{L}\right) \cong H_{1}\left(W_{0}\right)$. In this sense, an admissible homomorphism $\varphi$ always induces a covering $\left(W_{0}^{\varphi} ; X_{L}^{\varphi}, X_{H}^{\varphi}\right) \rightarrow\left(W ; X_{L}, X_{H}\right)$.

Proposition 4.3 Suppose $\left(W_{0} ; X_{L}, X_{H}\right)$ is a 1-solvable cobordism. Let $\left(W_{0}^{\varphi} ; X_{L}^{\varphi}, X_{H}^{\varphi}\right)$ be a covering induced from an admissible homomorphism $\varphi: H_{1}\left(M_{L}\right) \rightarrow \mathbb{Z} / p^{i} \oplus$ $\mathbb{Z} / p^{j}$, then

$$
P=\operatorname{Ker}\left(t H_{1}\left(X_{L}^{\varphi}, Y_{a}^{\varphi}\right) \rightarrow t H_{1}\left(W_{0}^{\varphi}, Y_{a}^{\varphi}\right)\right)
$$

is a metabolizer of the linking form $\lambda_{L}$.

Proof Suppose that we have the diagram (b) with two exact rows. Then,

$$
P^{\perp}=\left(\operatorname{Ad}\left(\lambda_{L}\right)\right)^{-1}\left(\operatorname{Ker} \partial^{\wedge}\right)=\operatorname{Ker}\left(\operatorname{inc}_{*}\right)=P .
$$

Hence, it suffices to prove the existence of diagram (b) with two exact rows.

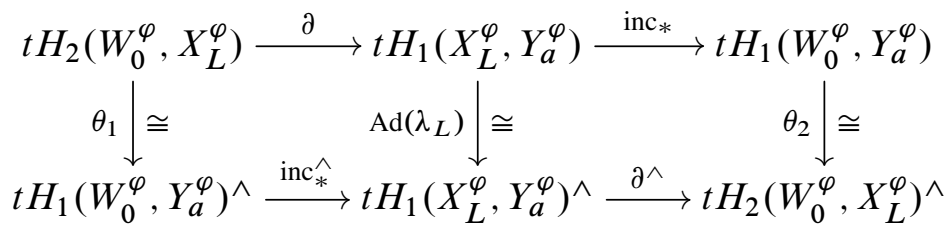

As in (a), $t H_{2}\left(W_{0}^{\varphi}, X_{L}^{\varphi}\right) \cong t H_{1}\left(W_{0}^{\varphi}, X_{H}^{\varphi}\right)^{\wedge}$ and $t H_{1}\left(W_{0}^{\varphi}, Y_{a}^{\varphi}\right) \cong t H_{2}\left(W_{0}^{\varphi}, \partial W_{0}^{\varphi}-\right.$ $\left.Y_{a}^{\varphi}\right)^{\wedge}$ by Poincaré duality and the universal coefficient theorem. (Note that $\partial W_{0}^{\varphi}=$ $X_{L}^{\varphi} \cup X_{H}^{\varphi}$.) So, from the following claim, we can define isomorphisms $\theta_{1}$ and $\theta_{2}$.

Claim The inclusion maps induce two isomorphisms:

(1) $t H_{1}\left(W_{0}^{\varphi}, Y_{a}^{\varphi}\right) \cong t H_{1}\left(W_{0}^{\varphi}, X_{H}^{\varphi}\right)$.

(2) $t H_{2}\left(W_{0}^{\varphi}, X_{L}^{\varphi}\right) \cong t H_{2}\left(W_{0}^{\varphi}, \partial W_{0}^{\varphi}-Y_{a}^{\varphi}\right)$. 
Proof of Claim By Proposition 3.2(3), $\left(W_{0}^{\varphi} ; X_{L}^{\varphi}, X_{H}^{\varphi}\right)$ is an $H_{1}$-cobordism with $\mathbb{Q}$-coefficients. From this and the proofs of [25, Lemmas 2.6, 2.7 and 2.9] ( $W_{0}$ plays the role of $E_{C}$ ),

$$
\text { Coker }\left(\operatorname{inc}_{*}: H_{1}\left(X_{H}^{\varphi}, Y_{a}^{\varphi}\right) \rightarrow H_{1}\left(W_{0}^{\varphi}, Y_{a}^{\varphi}\right)\right) \cong t H_{1}\left(W_{0}^{\varphi}, Y_{a}^{\varphi}\right) .
$$

From the homology long exact sequence of a triple $\left(W_{0}^{\varphi}, X_{H}^{\varphi}, Y_{a}^{\varphi}\right)$, we have an exact sequence

$$
0 \rightarrow t H_{1}\left(W_{0}^{\varphi}, Y_{a}^{\varphi}\right) \rightarrow H_{1}\left(W_{0}^{\varphi}, X_{H}^{\varphi}\right) \rightarrow H_{0}\left(X_{H}^{\varphi}, Y_{a}^{\varphi}\right)=0,
$$

which proves (1).

From the proof of [25, Lemma 2.5], $t H_{1}\left(\partial W_{0}^{\varphi}-Y_{a}^{\varphi}, X_{L}^{\varphi}\right)=0$ and the inclusion map $\left(\partial W_{0}^{\varphi}-Y_{a}^{\varphi}, X_{L}^{\varphi}\right) \rightarrow\left(\partial W_{0}^{\varphi}, X_{L}^{\varphi}\right)$ induces the zero map on $H_{2}$. In particular,

$$
\text { inc }_{*}: H_{2}\left(\partial W_{0}^{\varphi}-Y_{a}^{\varphi}, X_{L}^{\varphi}\right) \rightarrow H_{2}\left(W_{0}^{\varphi}, X_{L}^{\varphi}\right)
$$

is also the zero map. From the homology long exact sequence of a triple $\left(W_{0}^{\varphi}, \partial W_{0}^{\varphi}-\right.$ $\left.Y_{a}^{\varphi}, X_{L}^{\varphi}\right)$,

$$
H_{2}\left(W_{0}^{\varphi}, X_{L}^{\varphi}\right) \cong \operatorname{Ker}\left(\partial: H_{2}\left(W_{0}^{\varphi}, \partial W_{0}^{\varphi}-Y_{a}^{\varphi}\right) \rightarrow H_{1}\left(\partial W_{0}^{\varphi}-Y_{a}^{\varphi}, X_{L}^{\varphi}\right)\right) .
$$

By taking torsion subgroups, we obtain (2) via

$$
\begin{aligned}
t H_{2}\left(W_{0}^{\varphi}, X_{L}^{\varphi}\right) & \cong \operatorname{Ker}\left(t H_{2}\left(W_{0}^{\varphi}, \partial W_{0}^{\varphi}-Y_{a}^{\varphi}\right) \rightarrow t H_{1}\left(\partial W_{0}^{\varphi}-Y_{a}^{\varphi}, X_{L}^{\varphi}\right)=0\right) \\
& =t H_{2}\left(W_{0}^{\varphi}, \partial W_{0}^{\varphi}-Y_{a}^{\varphi}\right),
\end{aligned}
$$

completing the proof.

Commutativity of the diagram (b) also easily follows. For exactness of the first row of (b), we prove the following lemma.

Lemma 4.4 Suppose $\left(W_{0} ; X_{L}, X_{H}\right)$ is a 1 -solvable cobordism. We have the exact sequence

$$
t H_{2}\left(W_{0}^{\varphi}, X_{L}^{\varphi}\right) \stackrel{\partial}{\rightarrow} t H_{1}\left(X_{L}^{\varphi}, Y_{a}^{\varphi}\right) \stackrel{\text { inc }_{*}}{\rightarrow} t H_{1}\left(W_{0}^{\varphi}, Y_{a}^{\varphi}\right)
$$

which is the restriction of a long exact sequence of triple $\left(W_{0}^{\varphi}, X_{L}^{\varphi}, Y_{a}^{\varphi}\right)$ to their torsion subgroups.

Proof of Lemma 4.4 Since inc $_{*} \circ \partial=0$, we prove that $\operatorname{Ker}\left(\right.$ inc $\left._{*}\right) \subset \operatorname{Im} \partial$. Let $x \in \operatorname{Ker}\left(\right.$ inc $\left._{*}\right)$. By the homology long exact sequence of triple $\left(W_{0}^{\varphi}, X_{L}^{\varphi}, Y_{a}^{\varphi}\right)$, there 
exists $y \in H_{2}\left(W_{0}^{\varphi}, X_{L}^{\varphi}\right)$ such that $\partial y=x$. By Proposition 3.2(2), $F H_{2}\left(W_{0}^{\varphi}\right) \rightarrow$ $\mathrm{FH}_{2}\left(W_{0}^{\varphi}, X_{L}^{\varphi}\right)$ is surjective. Since it is equal to the composition

$$
F H_{2}\left(W_{0}^{\varphi}\right) \rightarrow F H_{2}\left(W_{0}^{\varphi}, Y_{a}^{\varphi}\right) \stackrel{j}{\rightarrow} F H_{2}\left(W_{0}^{\varphi}, X_{L}^{\varphi}\right),
$$

it follows that $j$ is surjective. We can choose $z \in F H_{2}\left(W_{0}^{\varphi}, Y_{a}^{\varphi}\right)$ such that $y-j(z) \in$ $t H_{2}\left(W_{0}^{\varphi}, X_{L}^{\varphi}\right)$. Then, $\partial(y-j(z))=\partial y=x$ and this shows $\operatorname{Ker}\left(\mathrm{inc}_{*}\right) \subset \operatorname{Im} \partial$.

Note that if $A \stackrel{f}{\rightarrow} B \stackrel{g}{\rightarrow} C$ is an exact sequence of abelian groups. Since $\mathbb{Q} / \mathbb{Z}$ is a divisible group, $\mathbb{Q} / \mathbb{Z}$ is an injective $\mathbb{Z}$-module. For any abelian group $G, \operatorname{Ext}_{\mathbb{Z}}(G, \mathbb{Q} / \mathbb{Z})=0$. Hence, $\operatorname{Hom}_{\mathbb{Z}}(-, \mathbb{Q} / \mathbb{Z})$ is an exact functor and we obtain $C^{\wedge} \rightarrow B^{\wedge} \rightarrow A^{\wedge}$ is exact. This proves that the second row of the diagram (b) is also exact and completes the proof of Proposition 4.3.

\subsection{Proof of Theorem A}

In this subsection, we prove Theorem A. Let $\left(W_{0} ; X_{L}, X_{H}\right)$ be a 1.5-solvable cobordism with $\beta_{2}\left(W_{0}, X_{L}\right)=2 r$. Note that $\partial W_{0}=X_{L} \cup \partial X_{H} \times I \cup-X_{H}=M_{L}$. Attach $X_{H} \times I$ to $W_{0}$ along $\partial X_{H} \times I$ to get

$$
W=W_{0} \cup_{\partial X_{H} \times I} X_{H} \times I
$$

with $\partial W=M_{L} \sqcup-M_{H}$. Recall that $\varphi: H_{1}\left(M_{L}\right) \rightarrow \mathbb{Z} / p^{i} \oplus \mathbb{Z} / p^{j}$. Applying a Mayer-Vietoris argument to $W=W_{0} \cup X_{H} \times I$, the inclusion induces an isomorphism $H_{1}\left(M_{L}\right) \cong H_{1}(W)$. So, $\varphi$ extends to $H_{1}(W)$ naturally and denote the induced cobordism of coverings by $\left(W^{\varphi} ; M_{L}^{\varphi}, M_{H}^{\varphi}\right)$.

From Proposition 4.3, we can take a metabolizer

$$
P:=\operatorname{Ker}\left(t H_{1}\left(X_{L}^{\varphi}, Y_{a}^{\varphi}\right) \rightarrow t H_{1}\left(W_{0}^{\varphi}, Y_{a}^{\varphi}\right)\right)
$$

of the linking form $\lambda_{L}$. We fix a character $\chi: H_{1}\left(M_{L}^{\varphi}\right) \rightarrow \mathbb{Z} / q^{k}$ satisfies that $\left.\chi\right|_{H_{1}\left(X_{L}^{\varphi}\right)}$ factors through

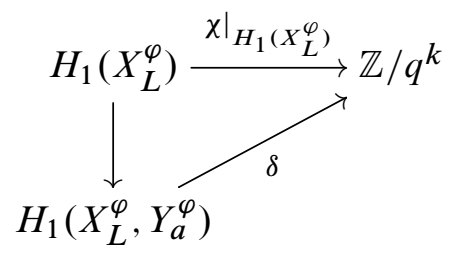

and $\delta$ vanishes on $P$. It remains to prove that $\tau(L, \chi)=0$. 
We have the following facts and remarks.

(1) From the arguments of [25, Propositions 2.10, 2.12] ( $W_{0}$ and $W$ play the role of $E_{C}$ and $W_{C}$, respectively), we have the following: if $\delta$ vanishes on $P$, then there exist an integer $l \geq k$ and a character $H_{1}\left(W^{\varphi}\right) \rightarrow \mathbb{Z} / q^{l}$, denoted by $\chi$ in an abuse of notation, which fits into the following diagram:

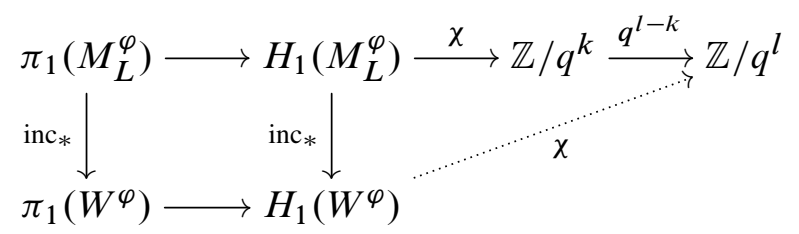

(2) Let $H_{1}\left(M_{L}\right)=\mathcal{H}^{\prime}$ and $\phi^{\prime}: \pi_{1}\left(M_{L}\right) \rightarrow H_{1}\left(M_{L}\right)$ be the Hurewicz homomorphism. Define $\phi: \pi_{1}\left(M_{L}^{\varphi}\right) \rightarrow \mathcal{H}^{\prime}$ to be the restriction of $\phi^{\prime}$ to the subgroup $\pi_{1}\left(M_{L}^{\varphi}\right)$. Let $\mathcal{H}=\operatorname{Im} \phi$. Since $H_{1}\left(M_{L}\right) \cong H_{1}(W), \phi^{\prime}$ extends to $\pi_{1}(W)$. Therefore, we use $\phi^{\prime}: \pi_{1}(W) \rightarrow \mathcal{H}^{\prime}$ and its restriction $\phi: \pi_{1}\left(W^{\varphi}\right) \rightarrow \mathcal{H}$ as an abuse of notation. Note that $\mathcal{H}^{\prime}$ is isomorphic to $\mathbb{Z}^{3}$ and $\mathcal{H}$ is also isomorphic to $\mathbb{Z}^{3}$ as a finite-index subgroup of $\mathcal{H}^{\prime}$.

(3) By (1) and (2), we have $\chi \times \phi: \pi_{1}\left(W^{\varphi}\right) \rightarrow \mathbb{Z} / q^{l} \times \mathcal{H}$. If we write $\mathcal{K}=\mathbb{C}(\mathcal{H})$, then $H_{*}\left(M_{L}^{\varphi} ; \mathcal{K}\right), H_{*}\left(W^{\varphi} ; \mathcal{K}\right)$, and $H_{*}\left(W^{\varphi}, M_{L}^{\varphi} ; \mathcal{K}\right)$ can be defined from

$\mathbb{Z}\left[\pi_{1}\left(W^{\varphi}\right)\right] \stackrel{\chi \times \phi}{\longrightarrow} \mathbb{Z}\left[\mathbb{Z} / q^{l} \times \mathcal{H}\right]=\mathbb{Z}\left[\mathbb{Z} / q^{l}\right][\mathcal{H}] \rightarrow \mathbb{Q}\left(\xi_{q^{l}}\right)(\mathcal{H}) \rightarrow \mathbb{C}(\mathcal{H})=\mathcal{K}$.

(4) By [25, Lemma 3.4], there is a 4 -manifold $W_{\chi}$ bounded by $2 q^{l}$ copies of a 3-torus $T^{3}$, which is over $\mathbb{Z} / q^{l} \times \mathcal{H}$ as follows:

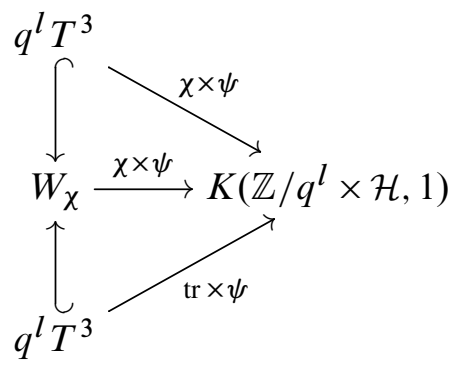

Here tr denotes the trivial character $\pi_{1}\left(T^{3}\right) \rightarrow \mathbb{Z} / q^{l}$ and $\psi: \pi_{1}\left(T^{3}\right) \cong \mathcal{H}$. Furthermore, the intersection forms of $W_{\chi}$ over $\mathbb{Q}$-coefficient and $\mathcal{K}$-coefficients are Witt-trivial.

We can attach $\chi \times \phi: q^{l} W^{\varphi} \rightarrow \mathbb{Z} / q^{l} \times \mathcal{H}$ and $W_{\chi}$ in (4) along $\chi \times \psi: q^{l} T^{3} \rightarrow$ $\mathbb{Z} / q^{l} \times \mathcal{H}$ to obtain the cobordism $\left(q^{l} W^{\varphi} \cup W_{\chi}, \chi \times \phi \cup \chi \times \psi\right)$ over $\mathbb{Z} / q^{l} \times \mathcal{H}$ 
between $q^{l}\left(M_{L}^{\varphi}, \chi \times \phi\right)$ and $-q^{l}\left(T^{3}, \operatorname{tr} \times \psi\right)$. From Definition 4.1, $\tau(L, \chi)=\left(\lambda_{\mathcal{K}}\left(q^{l} W^{\varphi} \cup W_{\chi}\right)-\mathcal{K} \otimes \lambda_{\mathbb{Q}}\left(q^{l} W^{\varphi} \cup W_{\chi}\right)\right) \otimes 1 / q^{l} \in L^{0}(\mathcal{K}) \otimes_{\mathbb{Z}} \mathbb{Z}[1 / q]$. By (4), $\left[\lambda_{\mathbb{Q}}\left(W_{\chi}\right)\right]=0 \in L^{0}(\mathbb{Q})$ and $\left[\lambda_{\mathcal{K}}\left(W_{\chi}\right)\right]=0 \in L^{0}(\mathcal{K})$. In the following two claims we will prove that $\left[\lambda_{\mathbb{Q}}\left(W^{\varphi}\right)\right]=0 \in L^{0}(\mathbb{Q})$ and $\left[\lambda_{\mathcal{K}}\left(W^{\varphi}\right)\right]=0 \in L^{0}(\mathcal{K})$. By Novikov additivity, these claims will complete the proof of Theorem A.

Claim 1 We have $\left[\lambda_{\mathbb{Q}}\left(W^{\varphi}\right)\right]=0 \in L^{0}(\mathbb{Q})$.

Proof of Claim 1 Applying the relative Mayer-Vietoris sequence (see for example Hatcher [27, page 152]), the sequence

$$
\rightarrow H_{i}\left(\partial X_{H} \times I, \partial X_{H}\right) \rightarrow H_{i}\left(W_{0}, X_{J}\right) \oplus H_{i}\left(X_{H} \times I, X_{H}\right) \rightarrow H_{i}\left(W, M_{J}\right) \rightarrow
$$

is exact. Since $H_{*}\left(\partial X_{H} \times I, \partial X_{H}\right)$ and $H_{*}\left(X_{H} \times I, X_{H}\right)$ vanish, $H_{i}\left(W_{0}, X_{J}\right) \cong$ $H_{i}\left(W, M_{J}\right)$. Similarly, $H_{i}\left(W_{0}^{\varphi}, X_{J}^{\varphi}\right) \cong H_{i}\left(W^{\varphi}, M_{J}^{\varphi}\right)$.

For brevity, let $A=\mathbb{Z} / p^{i} \oplus \mathbb{Z} / p^{j}$ and write $A=\left\{g_{1}, \ldots, g_{t}\right\}$. Since $\left(W_{0} ; X_{L}, X_{H}\right)$ is a 1.5-solvable cobordism, by Proposition 3.2(1) and (2),

$$
\beta_{2}\left(W^{\varphi}, M_{L}^{\varphi}\right)=\beta_{2}\left(W_{0}^{\varphi}, X_{L}^{\varphi}\right)=|A| \cdot \beta_{2}\left(W_{0}, X_{L}\right)=2 r t
$$

and inc $_{*}: H_{2}\left(W_{0}^{\varphi} ; \mathbb{Q}\right) \rightarrow H_{2}\left(W_{0}^{\varphi}, X_{J}^{\varphi} ; \mathbb{Q}\right)$ is surjective for $J=L, H$. Since we have $H_{2}\left(W_{0}^{\varphi}, X_{J}^{\varphi} ; \mathbb{Q}\right) \cong H_{2}\left(W^{\varphi}, M_{J}^{\varphi} ; \mathbb{Q}\right)$, inc $*: H_{2}\left(W^{\varphi} ; \mathbb{Q}\right) \rightarrow H_{2}\left(W^{\varphi}, M_{J}^{\varphi} ; \mathbb{Q}\right)$ is surjective, too. Applying Proposition 3.2(3), $H_{i}\left(W_{0}^{\varphi}, M_{J}^{\varphi} ; \mathbb{Q}\right)=0$ for $i=0,1$. From the homology long exact sequence of a pair $\left(W^{\varphi}, M_{J}^{\varphi}\right)$, this proves that $\left(W^{\varphi}, M_{L}^{\varphi}, M_{H}^{\varphi}\right)$ is an $H_{1}$-cobordism over $\mathbb{Q}$-coefficients.

Recall $\partial W=M_{L} \sqcup-M_{H}$. For $X=\partial W^{\varphi}, M_{L}^{\varphi}$, and $M_{H}^{\varphi}$, let

$$
I_{X}=\operatorname{Im}\left(\operatorname{inc}_{*}: H_{2}(X ; \mathbb{Q}) \rightarrow H_{2}\left(W^{\varphi} ; \mathbb{Q}\right)\right) .
$$

For $J=L, H$, using the homology long exact sequences of pairs,

$$
H_{2}\left(W^{\varphi} ; \mathbb{Q}\right) / I_{\partial W^{\varphi}} \cong H_{2}\left(W^{\varphi} ; \mathbb{Q}\right) / I_{M_{J}^{\varphi}} \cong H_{2}\left(W^{\varphi}, M_{J}^{\varphi} ; \mathbb{Q}\right)
$$

whose rank is $2 r t$. (A similar argument was used by Cochran and Kim in the proof of [14, Proposition 2.6].) We remark that to prove the last isomorphism, we used the fact that $\operatorname{inc}_{*}: H_{1}\left(M_{J}^{\varphi} ; \mathbb{Q}\right) \rightarrow H_{1}\left(W^{\varphi} ; \mathbb{Q}\right)$ is an isomorphism for $J=L, H$.

Let $l_{1}, \ldots, l_{r}, d_{1}, \ldots, d_{r}$ be (generators of) a 2 -Lagrangian and 1 -duals in $H_{2}\left(W^{\varphi} ; \mathbb{Z}\right)$. From the (right) group action of $A$ on $H_{2}\left(W^{\varphi} ; \mathbb{Z}\right)$, we define

$$
l_{i j}=l_{i} \cdot g_{j} \quad \text { and } \quad d_{k l}=d_{k} \cdot g_{l} \quad \text { for } 1 \leq i, k \leq r \text { and } 1 \leq j, l \leq t .
$$


The intersection pairing $\lambda_{\mathbb{Q}}\left(W^{\varphi}\right): H_{2}\left(W^{\varphi} ; \mathbb{Q}\right) / I_{\partial W^{\varphi}} \times H_{2}\left(W^{\varphi} ; \mathbb{Q}\right) / I_{\partial W^{\varphi}} \rightarrow \mathbb{Q}$ with respect to (the image of) $\left\{l_{i j}, d_{k l}\right\}$ is

$$
\left(\begin{array}{cc}
0 & I_{r t \times r t} \\
I_{r t \times r t} & X
\end{array}\right)
$$

because $l_{i} \cdot d_{k}$ is the Kronecker delta $\delta_{i k}$.

Let $L(\mathbb{Q}) \subset H_{2}\left(W^{\varphi} ; \mathbb{Q}\right) / I_{\partial W^{\varphi}}$ be the $\mathbb{Q}$-span of the image of $l_{i j} \otimes 1_{\mathbb{Q}}$. Then, $\lambda_{\mathbb{Q}}\left(W^{\varphi}\right)$ vanishes on $L(\mathbb{Q}) \times L(\mathbb{Q})$ and $\operatorname{dim}_{\mathbb{Q}} L(\mathbb{Q})=\frac{1}{2} \operatorname{dim}_{\mathbb{Q}}\left(H_{2}\left(W^{\varphi} ; \mathbb{Q}\right) / I_{\partial W^{\varphi}}\right)=$ $r t$. So, $\left[\lambda_{\mathbb{Q}}\left(W^{\varphi}\right)\right]=0 \in L^{0}(\mathbb{Q})$.

By [25, Lemma 3.2], $H_{*}\left(M_{J}^{\varphi} ; \mathcal{K}\right)=0$ for $J=H$ or $L$. Therefore, the twisted intersection form

$$
\lambda_{\mathcal{K}}\left(W^{\varphi}\right): H_{2}\left(W^{\varphi} ; \mathcal{K}\right) \times H_{2}\left(W^{\varphi} ; \mathcal{K}\right) \rightarrow \mathcal{K}
$$

is nonsingular.

Claim 2 We have $\left[\lambda_{\mathcal{K}}\left(W^{\varphi}\right)\right]=0 \in L^{0}(\mathcal{K})$.

Proof of Claim 2 Let $\alpha: \pi_{1}\left(W^{\varphi}\right) \stackrel{\chi}{\rightarrow} \mathbb{Z} / q^{l} \hookrightarrow \mathbb{C}^{\times}=\operatorname{GL}(1, \mathbb{C})$ and $\alpha^{\prime}: \pi_{1}(W) \rightarrow$ $\mathrm{GL}(t, \mathbb{C})$ be the induced representation of $\alpha$. Recall the maps $\phi^{\prime}: \pi_{1}(W) \rightarrow \mathcal{H}^{\prime}$ and $\phi: \pi_{1}\left(W^{\varphi}\right) \rightarrow \mathcal{H}$ in (2). Define $\Gamma:=\operatorname{Im}(\alpha \times \phi)$. There is a corresponding cover $\left(W^{\Gamma}, M_{L}^{\Gamma}\right) \rightarrow\left(W^{\varphi}, M_{L}^{\varphi}\right)$, where $\pi_{1}\left(W^{\Gamma}\right)=\operatorname{Ker}(\alpha \times \phi)$. Recall $W^{\varphi} \rightarrow W$ is $\mathbb{Z} / p^{i} \oplus \mathbb{Z} / p^{j}$-cover and $\alpha \times \phi: \pi_{1}\left(W^{\varphi}\right) \rightarrow \mathbb{C}^{\times} \times \mathcal{H}$. Since $\mathbb{Z} / p^{i} \oplus \mathbb{Z} / p^{j}, \mathbb{C}^{\times}$ and $\mathcal{H}$ are abelian,

$$
\pi_{1}(W)^{(2)} \leq \pi_{1}\left(W^{\varphi}\right)^{(1)} \leq \operatorname{Ker}(\alpha \times \phi)=\pi_{1}\left(W^{\Gamma}\right) .
$$

Equivalently, there is a sequence of coverings

$$
W^{(2)} \rightarrow W^{\Gamma} \rightarrow W^{\varphi} \rightarrow W .
$$

Since $\mathbb{Z} / q^{l} \hookrightarrow \mathbb{C}^{\times}$is injective, Ker $\alpha=\operatorname{Ker} \chi$, where $\alpha: \pi_{1}\left(W^{\varphi}\right) \stackrel{\chi}{\rightarrow} \mathbb{Z} / q^{l} \hookrightarrow \mathbb{C}^{\times}$. From this, $\Gamma \stackrel{\text { def }}{=} \operatorname{Im}(\alpha \times \phi)=\operatorname{Im}(\chi \times \phi)$. In particular, the ring homomorphism $\mathbb{Z}\left[\pi_{1}\left(W^{\varphi}\right)\right] \rightarrow \mathcal{K}$ in (3) factors through $\mathbb{Z} \Gamma$ and

$$
C_{*}\left(W^{\varphi} ; \mathcal{K}\right) \stackrel{\text { def }}{=} \mathcal{K} \otimes_{\mathbb{Z}\left[\pi_{1}\left(W^{\varphi}\right)\right]} C_{*}\left(W^{\varphi} ; \mathbb{Z}\left[\pi_{1} W^{\varphi}\right]\right)=\mathcal{K} \otimes_{\mathbb{Z} \Gamma} C_{*}\left(W^{\Gamma} ; \mathbb{Z}\right) .
$$

Choose 2-cycles $\left\{\tilde{l}_{1}, \ldots, \tilde{l}_{r}\right\} \subset C_{2}\left(W^{\Gamma} ; \mathbb{Z}\right)$ which represent the image of (generators of) the 2-Lagrangian under the map induced by $W_{0}^{(2)} \rightarrow W^{(2)} \rightarrow W^{\Gamma}$. The covering map $W^{\Gamma} \rightarrow W^{\varphi}$ induces a surjection $\operatorname{Cov}\left(W^{\Gamma} \mid W\right) \rightarrow \operatorname{Cov}\left(W^{\varphi} \mid W\right)=\left\{g_{1}, \ldots, g_{t}\right\}$. 
Choose a lift $\tilde{g}_{j} \in \operatorname{Cov}\left(W^{\Gamma} \mid W\right)$ of $g_{j}$ for each $j=1, \ldots, t$. From the right action of $\operatorname{Cov}\left(W^{\Gamma} \mid W\right)$ on $C_{2}\left(W^{\Gamma} ; \mathbb{Z}\right)$, define

$$
\tilde{l}_{i j}=\tilde{l}_{i} \cdot \tilde{g}_{j} \text { for } 1 \leq i \leq r \text { and } 1 \leq j \leq t .
$$

Let

$$
L(\mathcal{K}) \subset H_{2}\left(W^{\varphi} ; \mathcal{K}\right)=H_{2}\left(\mathcal{K} \otimes_{\mathbb{Z} \Gamma} C_{*}\left(W^{\Gamma} ; \mathbb{Z}\right)\right)
$$

be the $\mathcal{K}$-span of $\left\{\left[1_{\mathcal{K}} \otimes \tilde{l}_{i j}\right] \mid 1 \leq i \leq r, 1 \leq j \leq t\right\}$ in $H_{2}\left(W^{\varphi} ; \mathcal{K}\right)$. We remark that $L(\mathcal{K})$ does not depend on the choice of $\tilde{g}_{i}$. We claim that $L(\mathcal{K})$ is a Lagrangian for the nonsingular twisted intersection form $\lambda_{\mathcal{K}}\left(W^{\varphi}\right)$.

First, we prove $\lambda_{\mathcal{K}}$ vanishes on $L(\mathcal{K}) \times L(\mathcal{K})$. Since $\lambda_{\mathcal{K}}$ is $\mathcal{K}$-sesquilinear, the following is enough:

$$
\lambda_{\mathcal{K}}\left(\left[1_{\mathcal{K}} \otimes_{\mathbb{Z} \Gamma} \tilde{l}_{i k}\right],\left[1_{\mathcal{K}} \otimes_{\mathbb{Z} \Gamma} \tilde{l}_{j l}\right]\right)=\sum_{g \in \operatorname{Cov}(W \Gamma \mid W)} \lambda_{W \Gamma}\left(\tilde{l}_{i}, \tilde{l}_{j}\right) \tilde{g}_{j} g \tilde{g}_{k}^{-1}=0 .
$$

Now, we prove $\operatorname{dim}_{\mathcal{K}} L(\mathcal{K})=\frac{1}{2} \operatorname{dim}_{\mathcal{K}} H_{2}\left(W^{\varphi} ; \mathcal{K}\right)$. Recall that $H_{*}\left(M_{L} ; \mathcal{K}\right)=0$ by [25, Lemma 3.2]. Therefore, inc ${ }_{*}: H_{2}\left(W^{\varphi} ; \mathcal{K}\right) \rightarrow H_{2}\left(W^{\varphi}, M_{L}^{\varphi} ; \mathcal{K}\right)$ is an isomorphism. Now, for simplicity, we abuse notation by regarding $\widetilde{l}_{i j}$ as an element in $C_{2}\left(W^{\Gamma}, M_{L}^{\Gamma} ; \mathbb{Z}\right)$ and $L(\mathcal{K})$ as a subspace of $H_{2}\left(W^{\varphi}, M_{L}^{\varphi} ; \mathcal{K}\right)$.

Recall that $\left\{l_{1}, \ldots, l_{r}\right\}$ are the chosen generators of the 2-Lagrangian in $H_{2}\left(W^{\varphi} ; \mathbb{Z}\right)$. Since the covering $W^{\Gamma} \rightarrow W$ sends $\widetilde{g}_{j}$ to 1 , the image of $\left\{\left[\widetilde{l}_{i j}\right] \in H_{2}\left(W^{\Gamma} ; \mathbb{Z}\right) \mid\right.$ $1 \leq i \leq r, 1 \leq j \leq t\}$ in $H_{2}\left(W, M_{L} ; \mathbb{Z}\right)$ (via covering induced map) is exactly $\left\{\pi\left(l_{1}\right), \ldots, \pi\left(l_{r}\right)\right\}$ where $\pi: H_{2}\left(W^{\varphi}\right) \rightarrow H_{2}(W) \rightarrow H_{2}\left(W, M_{L}\right)$.

Since $\left(W_{0} ; X_{L}, X_{H}\right)$ is a 1.5 -solvable cobordism with $\beta_{2}\left(W_{0}, X_{L}\right)=2 r$, we have that $H_{2}\left(W, M_{L}\right) \cong H_{2}\left(W_{0}, X_{L}\right)$ is a free abelian group of rank $2 r$. Let

$$
L(\mathbb{Z} / q) \subset H_{2}\left(W, M_{L} ; \mathbb{Z} / q\right) \cong(\mathbb{Z} / q)^{2 r}
$$

be the $\mathbb{Z} / q$-span of $\left\{\pi\left(l_{i}\right) \otimes_{\mathbb{Z}} 1_{\mathbb{Z} / q}\right\}_{i=1}^{r}$. By the definition of a 2-Lagrangian, $\left\{\pi\left(l_{1}\right), \ldots, \pi\left(l_{r}\right)\right\}$ generates a rank $r$-summand of $H_{2}\left(W, M_{L}\right) \cong \mathbb{Z}^{2 r}$. In particular, from the universal coefficient theorem, $\operatorname{dim}_{\mathbb{Z} / q} L(\mathbb{Z} / q)=r$.

To apply Theorem 2.3, we fit our notation with that used in Section 2. Define $A=$ $\mathbb{Z} / p^{i} \oplus \mathbb{Z} / p^{j}, G=\pi_{1}(W), K=\pi_{1}\left(W^{\varphi}\right), C_{*}=C_{*}\left(W, M_{L} ; \mathbb{Z}\left[\pi_{1}(W)\right]\right), Q=\mathbb{C}$, $Q(\mathcal{H})=\mathcal{K}, d=1, \alpha \times \phi: \pi_{1}\left(W^{\varphi}\right) \rightarrow \mathbb{C}^{\times} \times \mathcal{H}$ and $\alpha^{\prime} \times \phi^{\prime}: \pi_{1}(W) \rightarrow \operatorname{GL}(t, \mathbb{C}) \times \mathcal{H}^{\prime}$. (As a $\mathbb{Z} K$-module, $C_{*}$ is isomorphic to $C_{*}\left(W^{\varphi}, M_{L}^{\varphi} ; \mathbb{Z}\left[\pi_{1}\left(W^{\varphi}\right)\right]\right)$.) We remark that we assumed in Section 2 that $\left.\alpha\right|_{\operatorname{Ker} \phi}$ factors through a $q$-group for some prime $q$. This is automatically satisfied for $\alpha: \pi_{1}\left(W^{\varphi}\right) \stackrel{\chi}{\rightarrow} \mathbb{Z} / q^{l} \hookrightarrow \mathbb{C}^{\times}$. 
With this notation, apply Theorem 2.3 for the case $I=\varnothing$ (that is, $M=\bar{M}=0$ ) and $n=0,1$ to obtain

$$
\operatorname{dim}_{\mathcal{K}} H_{n}\left(W^{\varphi}, M_{J}^{\varphi} ; \mathcal{K}\right) \leq \operatorname{dim}_{\mathbb{Z} / q} H_{n}\left(W, M_{J} ; \mathbb{Z} / q\right)=0
$$

for $n=0,1$ and $J=L$ or $H$. By duality and universal coefficient spectral sequence, $H_{i}\left(W^{\varphi}, M_{L}^{\varphi} ; \mathcal{K}\right)=0$ for $i=3,4$. From this,

$$
\operatorname{dim}_{\mathcal{K}} H_{2}\left(W^{\varphi}, M_{L}^{\varphi} ; \mathcal{K}\right)=\chi^{\mathcal{K}}\left(W^{\varphi}, M_{L}^{\varphi}\right)=\chi^{\mathbb{Q}}\left(W^{\varphi}, M_{L}^{\varphi}\right)=2 r t .
$$

The last equality is from $\beta_{2}\left(W^{\varphi}, M_{L}^{\varphi}\right)=2 r t$ and the fact that $\left(W^{\varphi} ; M_{L}^{\varphi}, M_{H}^{\varphi}\right)$ is an $H_{1}$-cobordism over $\mathbb{Q}$-coefficients. These are proved in the proof of Claim 1 .

Now, we apply Theorem 2.3 for the case $n=2, I=\{i \mid 1 \leq i \leq r\}$ and $x_{i}$ is a 2-cycle in $C_{*}$ such that

$\left[1_{\mathcal{K}} \otimes_{\mathbb{Z} \Gamma} \tilde{l}_{i}\right]=\left[1_{\mathcal{K}} \otimes_{\mathbb{Z} K} x_{i}\right] \in H_{2}\left(\mathcal{K} \otimes_{\mathbb{Z} K} C_{*}\right)=H_{2}\left(W^{\varphi}, M_{L}^{\varphi} ; \mathcal{K}\right) \quad$ for $i=1, \ldots, r$

Recall $\tilde{l}_{i j}=\tilde{l}_{i} \cdot \tilde{g}_{j}, \tilde{g}_{j} \in \operatorname{Cov}\left(W^{\Gamma} \mid W\right)$ is a lifting of $g_{j} \in \operatorname{Cov}\left(W^{\varphi} \mid W\right)$. Since $\operatorname{Cov}\left(W^{\varphi} \mid W\right)$ can be identified with the set of cosets of $K$ in $G$, by the definition in Theorem 2.3,

$$
M=\text { the } \mathcal{K} \text {-span of }\left\{\left[1_{\mathcal{K}} \otimes_{\mathbb{Z} \Gamma} \tilde{l}_{i j}\right] \mid 1 \leq i \leq r, 1 \leq j \leq t\right\}=L(\mathcal{K}) .
$$

Similarly, by the definition in Theorem $2.3, \bar{M}$ is the $\mathbb{Z} / q$-span of $\left\{\left[1_{\mathbb{Z} / q} \otimes_{\mathbb{Z} G} x_{i}\right]\right\}_{i=1}^{r}$. Since $\left\{\left[1_{\mathbb{Z} / q} \otimes_{\mathbb{Z} G} x_{i}\right]\right\}_{i=1}^{r}=\left\{1_{\mathbb{Z} / q} \otimes_{\mathbb{Z}} \pi\left(l_{i}\right)\right\}_{i=1}^{r}$,

$$
\bar{M}=\text { the } \mathbb{Z} / q-\text { span of }\left\{1_{\mathbb{Z} / q} \otimes_{\mathbb{Z}} \pi\left(l_{i}\right) \mid 1 \leq i \leq r\right\}=L(\mathbb{Z} / q) .
$$

From the conclusion of Theorem 2.3 for the above case, we have the inequality $\operatorname{dim}_{\mathcal{K}} H_{2}\left(W^{\varphi}, M_{L}^{\varphi} ; \mathcal{K}\right)-\operatorname{dim}_{\mathcal{K}} L(\mathcal{K})$

$$
\leq t \cdot\left(\operatorname{dim}_{\mathbb{Z} / q} H_{2}\left(W, M_{L} ; \mathbb{Z} / q\right)-\operatorname{dim}_{\mathbb{Z} / q} L(\mathbb{Z} / q)\right) ;
$$

that is,

$\operatorname{dim}_{\mathcal{K}} L(\mathcal{K}) \geq \operatorname{dim}_{\mathcal{K}} H_{2}\left(W, M_{L} ; \mathcal{K}\right)-t \cdot\left(\operatorname{dim}_{\mathbb{Z} / q} H_{2}\left(W, M_{L} ; \mathbb{Z} / q\right)-\operatorname{dim}_{\mathbb{Z} / q} L(\mathbb{Z} / q)\right)$

$$
=2 r t-r t+r t=r t \text {. }
$$

On the other hand, $\operatorname{dim}_{\mathcal{K}} L(\mathcal{K}) \leq r t$ because $L(\mathcal{K})$ is the $\mathcal{K}$-span of $r t$ elements. So, $\operatorname{dim}_{\mathcal{K}} L(\mathcal{K})=r t=\frac{1}{2} \operatorname{dim}_{\mathcal{K}} H_{2}\left(W^{\varphi} ; \mathcal{K}\right)$ and $L(\mathcal{K})$ is a Lagrangian of $\lambda_{\mathcal{K}}\left(W^{\varphi}\right)$. Therefore, $\left[\lambda_{\mathcal{K}}\left(W^{\varphi}\right)\right]=0 \in L^{0}(\mathcal{K})$. 


\section{Solvable cobordism and abelian invariants of links}

In this section, we study the abelian invariants of links (studied in $[28 ; 30]$ ) in the context of Whitney tower/grope concordance using $h$-solvable cobordism. Throughout this section, $\mu$ is the fixed natural number. Denote $\mathbb{Z}\left[t_{1}^{ \pm}, \ldots, t_{\mu}^{ \pm}\right]$by $\Lambda_{\mu}$. The ring $\Lambda_{\mu}$ is endowed with the involution $-: t_{i} \mapsto t_{i}^{-1}$. Let $S$ be the multiplicative set generated by $\left\{t_{1}-1, \ldots, t_{\mu}-1\right\}$. Denote the localization of $\Lambda_{\mu}$ with respect to $S$ by $\Lambda_{\mu} S$. Let $\mathcal{K}$ be the quotient field of $\Lambda_{\mu}$.

\subsection{Blanchfield form of $\mu$-component links}

Let $R$ be a unique factorization domain with an involution - and quotient field $K$ (our case is $\left.R=\Lambda_{\mu S}, K=\mathcal{K}\right)$. We recall the definition of the Witt group $W(K, R,-)$.

A linking pairing over $R$ is an $R$-module $M$ with a sesquilinear pairing

$$
b: M \times M \rightarrow K / R
$$

such that for all $x, y, z \in M$ and $r \in R$,

(1) $b(x, y+z)=b(x, y)+b(x, z)$,

(2) $b(r x, y)=r b(x, y)=b(x, \bar{r} y)$,

(3) $b(x, y)=\overline{b(y, x)}$.

(Here, the involution - on $K / R$ is induced from the involution on $R$.) We denote it by $(M, b)$ or just $b$ when $M$ is clearly understood. A linking pairing $(M, b)$ is primitive (nonsingular) if the adjoint of $b$,

$$
\operatorname{Ad}(b): M \rightarrow \operatorname{Hom}_{R}(M, K / R)
$$

is an injection (an $R$-module isomorphism), respectively. The sum of linking pairings $(M, b)$ and $\left(M^{\prime}, b^{\prime}\right)$ is $\left(M \oplus M^{\prime}, b \oplus b^{\prime}\right)$. A pairing $(M, b)$ is neutral if there is a submodule $N$ of $M$ such that

$$
N=N^{\perp}=\{m \in M \mid b(n, m)=0 \text { for all } n \in N\} .
$$

Two pairings $(M, b)$ and $\left(M^{\prime}, b^{\prime}\right)$ are Witt equivalent if there are neutral pairings $(N, c)$ and $\left(N^{\prime}, c^{\prime}\right)$ such that $(M, b) \oplus(N, c) \cong\left(M^{\prime}, b^{\prime}\right) \oplus\left(N^{\prime}, c^{\prime}\right)$. Then, the set of Witt equivalence classes of linking pairings over $R$ with an involution - is an abelian group, denoted by $W(K, R,-)$.

For an $R$-module $M$, following [28, Chapter 3], we define the $R$-torsion submodule of $M$,

$$
t M=\{m \in M \mid r m=0 \text { for some } r \neq 0 \in R\}=\operatorname{Ker}\left(M \rightarrow M \otimes_{R} K\right),
$$


the maximal pseudonull submodule of $M$,

$$
\begin{gathered}
z M=\operatorname{Ker}\left(t M \rightarrow \operatorname{Ext}_{R}^{1}\left(\operatorname{Ext}_{R}^{1}(t M, R), R\right)\right), \\
\hat{t} M=t M / z M .
\end{gathered}
$$

A torsion $R$-module $M$ is pseudonull if $M_{\mathfrak{p}}=0$ for every height- 1 prime ideal $\mathfrak{p}$ of $R$ where $M_{\mathfrak{p}}$ is the localization of $M$ with respect to the multiplicative set $M-\mathfrak{p}$. Note that a submodule and a quotient module of a pseudonull module are pseudonull. The following observation will be useful.

Lemma 5.1 Suppose that

$$
A \stackrel{f}{\rightarrow} B \stackrel{g}{\rightarrow} C \stackrel{h}{\rightarrow} D
$$

is an exact sequence of $R$-modules and $A=t A$ and $D=t D$. Then,

$$
t A \stackrel{f \mid}{\longrightarrow} t B \stackrel{g \mid}{\rightarrow} t C \stackrel{h \mid}{\rightarrow} t D
$$

is also exact. (Here, $f|, g|$, and $h \mid$ are the restriction of $f, g$ and $h$ to their $R$-torsion submodules, respectively.)

Proof Since $t A=A$ and $A \stackrel{f}{\rightarrow} B \stackrel{g}{\rightarrow} C$ is exact, $\operatorname{Im} f \mid=\operatorname{Im} f=\operatorname{Ker} g$. Then, Ker $g \mid=$ Ker $g \cap t B=\operatorname{Im} f|\cap t B=\operatorname{Im} f|$ since $\operatorname{Im} f \mid \subset t B$.

Since $B \stackrel{g}{\rightarrow} C \stackrel{h}{\rightarrow} D$ is exact, Ker $h \mid=\operatorname{Ker} h \cap t C=\operatorname{Im} g \cap t C$. Suppose that $x \in B$ and $g(x) \in t C$. Then, there exists $r \in R-\{0\}$ such that $g(r x)=r g(x)=0$. Since $A=t A$ and $D=t D$, we have the commutative diagram

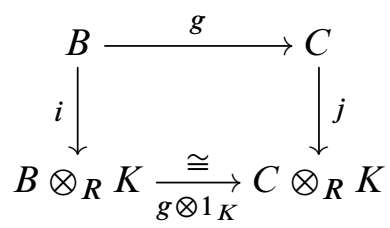

where $i$ and $j$ are natural maps. Then, $g \otimes 1_{K} \circ i(r x)=j \circ g(r x)=0$. Since $g \otimes 1_{K}$ is injective, $r x \in \operatorname{Ker} i=t B$. There exists $s \in R-\{0\}$ such that $s r x=0$. Therefore, $g(x) \in \operatorname{Im} g \mid$ and $\operatorname{Ker} h|=\operatorname{Im} g \cap t C \subset \operatorname{Im} g|$. Since $h \circ g=0, \operatorname{Im} g|\subset \operatorname{Ker} h|$. This completes the proof.

Let $L$ be a $\mu$-component link and $X_{L}$ be the link exterior of $L$. From Alexander duality, the Hurewicz map becomes $\pi_{1}\left(X_{L}\right) \rightarrow H_{1}\left(X_{L}\right)=\mathbb{Z}^{\mu}$. We have the exact sequence

$$
H_{1}\left(\partial X_{L} ; \Lambda_{\mu}\right) \rightarrow H_{1}\left(X_{L} ; \Lambda_{\mu}\right) \rightarrow H_{1}\left(X_{L}, \partial X_{L} ; \Lambda_{\mu}\right) \rightarrow H_{0}\left(\partial X_{L} ; \Lambda_{\mu}\right)
$$


whose extremal terms are $\prod_{i=1}^{\mu}\left(t_{i}-1\right)$-torsion (in particular, $S$-torsion) because $\mathbb{Z}^{\mu}$ cover of $\partial X_{L}$ is a disjoint union of $S^{1} \times \mathbb{R}$ or $\mathbb{R} \times \mathbb{R}$. From this observation, by localizing the above sequence with respect to $S$, we obtain $H_{1}\left(X_{L} ; \Lambda_{\mu S}\right) \cong H_{1}\left(X_{L}, \partial X_{L} ; \Lambda_{\mu S}\right)$. It follows from the (localized) Blanchfield duality [1] (as in [28, page 36]) that we have the following primitive linking pairing:

$$
b_{L}: \hat{t} H_{1}\left(X_{L} ; \Lambda_{\mu S}\right) \times \hat{t} H_{1}\left(X_{L} ; \Lambda_{\mu S}\right) \rightarrow \mathcal{K} / \Lambda_{\mu S} .
$$

Here, to define $b_{L}$, we need the fact that $\mathcal{K} / \Lambda_{\mu S}$ contains no nontrivial pseudonull submodule, [28, Theorem 3.9(2)].

In this setting, Hillman [28, Theorem 2.4] proved $\left[b_{L}\right] \in W\left(\mathcal{K}, \Lambda_{\mu S},-\right)$ is a concordance invariant of $L$. Here is our theorem which generalizes [28, Theorem 2.4].

Theorem B Suppose $L_{0}$ and $L_{1}$ are $\mu$-component links. If two link exteriors $X_{L_{0}}$ and $X_{L_{1}}$ are 1-solvable cobordant, then $\left[b_{L_{0}}\right]=\left[b_{L_{1}}\right] \in W\left(\mathcal{K}, \Lambda_{\mu S},-\right)$. In particular, the conclusion holds if $L_{0}$ and $L_{1}$ are height-3 Whitney tower/grope concordant.

Proof We proceed similarly to Hillman's proof of [28, Theorem 2.4], using a 1solvable cobordism instead of a concordance exterior. For this purpose, we need the following fact from [8]. Suppose $W$ is a 1-solvable cobordism between $X_{L_{0}}$ and $X_{L_{1}}$.

Lemma 5.2 (A special case of [8, Corollary 4.14]) The sequence

$$
t H_{2}\left(W, \partial W ; \Lambda_{\mu}\right) \stackrel{\partial}{\rightarrow} t H_{1}\left(\partial W ; \Lambda_{\mu}\right) \stackrel{i_{*}}{\rightarrow} t H_{1}\left(W ; \Lambda_{\mu}\right)
$$

is exact.

In fact, Lemma 5.2 is obtained from [8, Corollary 4.14] by setting $n=1, G=\mathbb{Z}^{\mu}$, $\phi: \pi_{1}(W) \rightarrow H_{1}(W)=\mathbb{Z}^{\mu}$, and $R=\mathbb{Z}$.

Using Lemma 5.2 in place of the exact sequence in [28, page 39], we can carry out the arguments in [28, page 39-40] for a 1-solvable cobordism $W$. For convenience of the readers, we present details below.

Note that

$$
\partial W=X_{L_{0}} \cup \mu\left(S^{1} \times S^{1} \times I\right) \cup-X_{L_{1}}
$$

and $\mathbb{Z}^{\mu}=H_{1}\left(X_{L_{i}}\right) \stackrel{\text { inc }^{*}}{\longrightarrow} H_{1}(W)$ is an isomorphism for $i=0,1$.

By the $\left(\Lambda_{\mu S}\right.$-coefficient) Mayer-Vietoris sequence of the triple $\left(\partial W, X_{L_{0}}, X_{L_{1}}\right)$,

$$
H_{1}\left(\partial W ; \Lambda_{\mu S}\right) \cong H_{1}\left(X_{L_{0}} ; \Lambda_{\mu S}\right) \oplus H_{1}\left(X_{L_{1}} ; \Lambda_{\mu S}\right),
$$


since $H_{i}\left(\mu\left(S^{1} \times S^{1} \times I\right) ; \Lambda_{\mu}\right)$ is $S$-torsion for $i=0,1$. From this, the (localized) Blanchfield form

$$
b_{\partial W}: \hat{t} H_{1}\left(\partial W ; \Lambda_{\mu S}\right) \times \hat{t} H_{1}\left(\partial W ; \Lambda_{\mu S}\right) \rightarrow \mathcal{K} / \Lambda_{\mu S}
$$

is the direct sum $b_{L_{0}} \oplus\left(-b_{L_{1}}\right)$. If we show $b_{\partial W}$ is neutral, then $\left[b_{L_{0}}\right]=\left[b_{L_{1}}\right] \in$ $W\left(\mathcal{K}, \Lambda_{\mu S},-\right)$ because $b_{L_{0}} \oplus\left(b_{L_{1}} \oplus\left(-b_{L_{1}}\right)\right)=b_{L_{1}} \oplus b_{\partial W}$. Therefore, it suffices to find a submodule $P_{0}$ of $\hat{t} H_{1}\left(\partial W ; \Lambda_{\mu S}\right)$ such that $P_{0}=P_{0}^{\perp}$.

Let $I_{\partial W}$ and $I_{W}$ be $\Lambda_{\mu S}$-coefficient intersection forms of $\partial W$ and $W$, respectively. We have Blanchfield form,

$$
b_{W}: \hat{t} H_{1}\left(W ; \Lambda_{\mu S}\right) \times \hat{t} H_{2}\left(W, \partial W ; \Lambda_{\mu S}\right) \rightarrow \mathcal{K} / \Lambda_{\mu S} .
$$

Let $\pi: t H_{1}\left(\partial W ; \Lambda_{\mu S}\right) \rightarrow \widehat{t} H_{1}\left(\partial W ; \Lambda_{\mu S}\right)$ be the quotient map. Let

$$
i_{\#}: C_{*}\left(\partial W ; \Lambda_{\mu S}\right) \rightarrow C_{*}\left(W ; \Lambda_{\mu S}\right) \quad \text { and } \quad i_{*}: t H_{1}\left(\partial W ; \Lambda_{\mu S}\right) \rightarrow t H_{1}\left(W ; \Lambda_{\mu S}\right)
$$

be the inclusion induced maps. Let

$$
P=\operatorname{Im}\left(t H_{2}\left(W, \partial W ; \Lambda_{\mu S}\right) \stackrel{\partial}{\rightarrow} t H_{1}\left(\partial W ; \Lambda_{\mu S}\right) \stackrel{\pi}{\rightarrow} \hat{t} H_{1}\left(\partial W ; \Lambda_{\mu S}\right)\right) .
$$

By Lemma 5.2, $P=\pi\left(\operatorname{Ker} i_{*}\right)$. We will prove that $P^{\perp}=P^{\perp \perp}$.

First, we prove $P \leq P^{\perp}$. Choose relative 2 -cycles $Q$ and $R$ in $C_{2}\left(W, \partial W ; \Lambda_{\mu S}\right)$ representing the classes in $t H_{2}\left(W, \partial W ; \Lambda_{\mu S}\right)$. Denote the boundaries of $Q$ and $R$ by $q, r \in C_{1}\left(\partial W ; \Lambda_{\mu S}\right)$, respectively. The corresponding classes $[q],[r]$ in $\hat{t} H_{1}\left(\partial W ; \Lambda_{\mu S}\right)$ are actually in $P$. There exists $a \in \Lambda_{\mu S}-\{0\}$ such that $a q$ is the boundary of a 2-chain $u \in C_{2}\left(\partial W ; \Lambda_{\mu S}\right)$. Then

$b_{\partial W}([q],[r])=a^{-1} I_{\partial W}(u, r)=-a^{-1} I_{W}\left(i_{\#}(u), R\right)=-b_{W}\left(\left[i_{\#}(q)\right],[R]\right)\left(\bmod \Lambda_{\mu S}\right)$.

Since $q$ is the boundary of the relative 2 -cycle $Q,\left[i_{\#}(q)\right]=0 \in \hat{t} H_{1}\left(W ; \Lambda_{\mu S}\right)$. Therefore

$$
b_{\partial W}([q],[r])=0 \quad \text { for all }[q],[r] \in P .
$$

This shows that $P \leq P^{\perp}$.

Suppose that $x \in C_{1}\left(\partial W ; \Lambda_{\mu S}\right)$ represents a class in $t H_{1}\left(\partial W ; \Lambda_{\mu S}\right)$ and $[x] \in P^{\perp}$. That is,

$$
b_{\partial W}([x],[y])=0
$$

for all $y$ which is the boundary of a relative 2-cycle $Y \in C_{2}\left(W, \partial W ; \Lambda_{\mu S}\right)$. Therefore

$$
b_{W}\left(\left[i_{\#}(x)\right],[Y]\right)=-b_{\partial W}([x],[y])=0 \quad \text { for all }[Y] \in \hat{t} H_{2}\left(W, \partial W ; \Lambda_{\mu S}\right) .
$$


By Blanchfield duality for $(W, \partial W)$, the adjoint of $b_{W}$,

$$
\operatorname{Ad}\left(b_{W}\right): \hat{t} H_{1}\left(W ; \Lambda_{\mu S}\right) \rightarrow \operatorname{Hom}_{\Lambda_{\mu S}}\left(\hat{t} H_{2}\left(W, \partial W ; \Lambda_{\mu S}\right), K / \Lambda_{\mu S}\right)
$$

is injective. Therefore, $\left[i_{\#}(x)\right]=0 \in \hat{t} H_{1}\left(W ; \Lambda_{\mu S}\right)$ or $i_{\#}(x)$ represents a homology class in $z H_{1}\left(W ; \Lambda_{\mu S}\right)$. The above argument shows $i_{*}\left(\pi^{-1}\left(P^{\perp}\right)\right)$ is pseudonull as a submodule of $z H_{1}\left(W ; \Lambda_{\mu S}\right)$. Since $P=\pi\left(\operatorname{Ker} i_{*}\right) \leq P^{\perp}, \operatorname{Ker} i_{*} \subset \pi^{-1}\left(P^{\perp}\right)$. Thus, $\pi^{-1}\left(P^{\perp}\right) / \operatorname{Ker} i_{*} \cong i_{*}\left(\pi^{-1}\left(P^{\perp}\right)\right)$ is pseudonull, and therefore, $P^{\perp} / P=$ $\pi\left(\pi^{-1}\left(P^{\perp}\right)\right) / \pi\left(\operatorname{Ker} i_{*}\right)$ is also pseudonull. Since $\mathcal{K} / \Lambda_{\mu S}$ has no nontrivial pseudonull submodule and $P^{\perp} / P$ is pseudonull,

$P^{\perp}=\left\{x \mid b_{\partial W}(x, y)=0\right.$ for all $\left.y \in P\right\}=\left\{x \mid b_{\partial W}(x, y)\right.$ for all $\left.y \in P^{\perp}\right\}=P^{\perp \perp}$.

This completes the proof.

\subsection{Multivariable Alexander polynomial of links}

In this subsection, we prove Theorem $\mathrm{C}$ which generalizes [30, Theorems A, B] concerning the Fox-Milnor condition for the Alexander polynomial of links.

First, we recall some definitions of [30]. Since $\Lambda_{\mu}$ is Nötherian, for a finitely generated $\Lambda_{\mu}$-module $M$, we can choose a presentation matrix $P$ of $M$ from an exact sequence

$$
\Lambda_{\mu}^{m} \stackrel{P}{\rightarrow} \Lambda_{\mu}^{n} \rightarrow M \rightarrow 0 .
$$

Moreover, for all $k$, one can choose a $m \times n$ presentation matrix $P$ with $n>k$ and $m \geq n-k$. In this situation, define the $k^{\text {th }}$ Alexander polynomial of $M$, denoted by $\Delta_{k}(M)$, to be the greatest common divisor of the size $(n-k) \times(n-k)$ minors of $P$. (It is well known that $\Delta_{k}(M)$ is well defined up to a unit of $\Lambda_{\mu}$ which is proved by Crowell and Fox in [20].)

Remark 5.3 (1) From [1, Theorem 4.10], if $d=\operatorname{rank}_{\Lambda_{\mu}} M$, then $\Delta_{d}(M)=$ $\Delta_{0}(t M)$.

(2) From [30, Lemma 2.4], if $0 \rightarrow M^{\prime} \rightarrow M \rightarrow M^{\prime \prime} \rightarrow 0$ is a short exact sequence of $\Lambda_{\mu}$-torsion modules, then $\Delta_{0}(M)=\Delta_{0}\left(M^{\prime}\right) \Delta_{0}\left(M^{\prime \prime}\right)$. More generally, if

$$
0 \rightarrow M_{1} \rightarrow M_{2} \rightarrow \cdots \rightarrow M_{r} \rightarrow 0
$$

is exact, then $\prod_{i=1}^{r} \Delta_{0}\left(M_{i}\right)^{(-1)^{r}}=1$.

(3) Suppose that $M_{1} \stackrel{f_{1}}{\longrightarrow} M_{2} \stackrel{f_{2}}{\longrightarrow} M_{3} \stackrel{f_{3}}{\longrightarrow} M_{4}$ is an exact sequence of $\Lambda_{\mu}$-torsion modules. Then $0 \rightarrow \operatorname{Im} f_{1} \rightarrow M_{2} \rightarrow M_{3} \rightarrow \operatorname{Im} f_{3} \rightarrow 0$ is exact. By (2),

$$
\Delta_{0}\left(M_{2}\right) \Delta_{0}\left(\operatorname{Im} f_{3}\right)=\Delta_{0}\left(M_{3}\right) \Delta_{0}\left(\operatorname{Im} f_{1}\right) \text {. }
$$

Here $\Delta_{0}\left(\operatorname{Im} f_{1}\right)$ and $\Delta_{0}\left(\operatorname{Im} f_{3}\right)$ divide $\Delta_{0}\left(M_{1}\right)$ and $\Delta_{0}\left(M_{4}\right)$, respectively. 
Recall that $L$ is a $\mu$-component link in $S^{3}$ and the meridian map is $\pi_{1}\left(X_{L}\right) \rightarrow \mathbb{Z}^{\mu}$. We define the torsion Alexander polynomial of $L$ by $\Delta_{L}^{T}:=\Delta_{0}\left(t H_{1}\left(X_{L} ; \Lambda_{\mu}\right)\right)$. Now we state our theorem.

Theorem C Suppose $L_{0}$ and $L_{1}$ are $\mu$-component links. If two link exteriors $X_{L_{0}}$ and $X_{L_{1}}$ are 1-solvable cobordant, then:

(1) $\operatorname{rank}_{\Lambda_{\mu}} H_{1}\left(X_{L_{0}} . \Lambda_{\mu}\right)=\operatorname{rank}_{\Lambda_{\mu}} H_{1}\left(X_{L_{1}} ; \Lambda_{\mu}\right)$.

$$
\Delta_{L_{0}}^{T} f_{0} \overline{f_{0}} \doteq \Delta_{L_{1}}^{T} f_{1} \bar{f}_{1}
$$

for some $f_{i}\left(t_{1}, \ldots, t_{\mu}\right) \in \Lambda_{\mu}$, with $i=0,1$ and with $\left|f_{i}(1, \ldots, 1)\right|=1$.

In particular, the conclusion holds if $L_{0}$ and $L_{1}$ are height-3 Whitney tower/grope concordant.

To prove Theorem $\mathrm{C}$, we need to prove the following generalization of [30, Lemma 2.1].

Lemma 5.4 Let $X$ be a finite connected $C W$-complex with an epimorphism

$$
\gamma: \pi_{1}(X) \rightarrow \mathbb{Z}^{\mu}
$$

Let $X_{0}$ be a subcomplex of $X$. For some fixed $k$, if $H_{k}\left(X, X_{0} ; \mathbb{Z}\right)=\mathbb{Z}^{l}$ and

$$
\operatorname{rank}_{\Lambda_{\mu}} H_{k}\left(X, X_{0} ; \Lambda_{\mu}\right)=l
$$

then the $l^{\text {th }}$ Alexander polynomial $A=\Delta_{l}\left(H_{k}\left(X, X_{0} ; \Lambda_{\mu}\right)\right)=\Delta_{0}\left(t H_{k}\left(X, X_{0} ; \Lambda_{\mu}\right)\right)$ satisfies $|A(1, \ldots, 1)|=1$.

Remark For the $l=0$ case (see [30, Lemma 2.1]), we only need to assume that $H_{k}\left(X, X_{0} ; \mathbb{Z}\right)=0$ because from our proof, we can deduce

$$
\operatorname{rank}_{\Lambda_{\mu}} H_{k}\left(X, X_{0} ; \Lambda_{\mu}\right) \leq 0 .
$$

In this sense, Lemma 5.4 is a generalization of [30, Lemma 2.1].

Proof of Lemma 5.4 Since $X_{0}$ is a subcomplex of $X$, for all $q$, we fix a basis for the $q^{\text {th }}$ (cellular) chain complex $C_{q}\left(X, X_{0} ; \mathbb{Z}\right) \cong \mathbb{Z}^{s_{q}}$. By lifting each element in the chosen bases, we also fix a $\Lambda_{\mu}$-basis for the $C_{q}\left(X, X_{0} ; \Lambda_{\mu}\right)$ for all $q$. With these chosen bases, we can write $\partial_{q}: C_{q}\left(X, X_{0} ; \Lambda_{\mu}\right) \rightarrow C_{q-1}\left(X, X_{0} ; \Lambda_{\mu}\right)$ as a matrix $\left(\alpha_{i j}^{q}\right)$, $\alpha_{i j}^{q} \in \Lambda_{\mu}$.

With respect to the chosen basis of $C_{*}\left(X, X_{0} ; \mathbb{Z}\right), \partial_{q}: C_{q}\left(X, X_{0} ; \mathbb{Z}\right) \rightarrow C_{q-1}\left(X, X_{0} ; \mathbb{Z}\right)$ is represented by the integral matrix $\left(\alpha_{i j}^{q}(1, \ldots, 1)\right)$. Let $\widetilde{r}_{q}=\operatorname{rank}\left(\alpha_{i j}^{q}\right), r_{q}=$ $\operatorname{rank}\left(\alpha_{i j}^{q}(1, \ldots, 1)\right)$. Then $r_{q} \leq \widetilde{r}_{q}$. Since $H_{k}\left(X, X_{0} ; \mathbb{Z}\right)=\mathbb{Z}^{l}$,

$$
l=\operatorname{rank}_{\mathbb{Z}} \operatorname{Ker} \partial_{k}-\operatorname{rank}_{\mathbb{Z}} \operatorname{Im} \partial_{k+1}=s_{k}-r_{k}-r_{k+1} .
$$


Similarly, from $\operatorname{rank}_{\Lambda_{\mu}} H_{k}\left(X, X_{0} ; \Lambda_{\mu}\right)=l$,

$$
l=s_{k}-\widetilde{r}_{k}-\widetilde{r}_{k+1} .
$$

Since $r_{q} \leq \widetilde{r}_{q}$ for all $q$,

$$
l=s_{k}-\tilde{r}_{k}-\tilde{r}_{k+1} \leq s_{k}-r_{k}-r_{k+1}=l
$$

which implies that $r_{k}=\widetilde{r}_{k}, r_{k+1}=\widetilde{r}_{k+1}$.

Since Coker $\partial_{k+1}=C_{k}\left(X, X_{0} ; \Lambda_{\mu}\right) / \operatorname{Im} \partial_{k+1}$ and $\operatorname{Im} \partial_{k} \cong C_{k}\left(X, X_{0} ; \Lambda_{\mu}\right) / \operatorname{Ker} \partial_{k}$, we have the short exact sequence

$$
0 \rightarrow H_{k}\left(X, X_{0} ; \Lambda_{\mu}\right) \rightarrow \text { Coker } \partial_{k+1} \rightarrow \operatorname{Im} \partial_{k} \rightarrow 0 .
$$

As a submodule of a free module, $\operatorname{Im} \partial_{k}$ is a $\Lambda_{\mu}$-torsion free module of rank $\widetilde{r}_{k}=r_{k}$. Then $t H_{k}\left(X, X_{0} ; \Lambda_{\mu}\right)=t$ Coker $\partial_{k+1}$ and $\operatorname{dim}_{\mathcal{K}} \operatorname{Coker} \partial_{k+1} \otimes_{\Lambda_{\mu}} \mathcal{K}=l+r_{k}$ :

$$
\begin{aligned}
\Delta_{l}\left(H_{k}\left(X, X_{0} ; \Lambda_{\mu}\right)\right)=\Delta_{0}\left(t H_{k}\left(X, X_{0} ; \Lambda_{\mu}\right)\right) & =\Delta_{0}\left(t \text { Coker } \partial_{k+1}\right) \\
& =\Delta_{l+r_{k}}\left(\text { Coker } \partial_{k+1}\right) .
\end{aligned}
$$

The first and last equalities follow from Remark 5.3(1). Similarly, we have the short exact sequence

$$
0 \rightarrow H_{k}\left(X, X_{0} ; \mathbb{Z}\right) \rightarrow \operatorname{Coker} \partial_{k+1}^{\mathbb{Z}} \rightarrow \operatorname{Im} \partial_{k}^{\mathbb{Z}} \rightarrow 0 .
$$

(Here, to avoid confusion, we denote the differential on $C_{*}\left(X, X_{0} ; \mathbb{Z}\right)$ by $\partial_{*}^{\mathbb{Z}}$.) As a subgroup of a finitely generated free abelian group, $\operatorname{Im} \partial_{k}^{\mathbb{Z}}$ is a free abelian group of rank $r_{k}$. Therefore

$$
\text { Coker } \partial_{k+1}^{\mathbb{Z}}=H_{k}\left(X, X_{0} ; \mathbb{Z}\right) \oplus \mathbb{Z}^{r_{k}}=\mathbb{Z}^{l+r_{k}}
$$

(Here we used the assumption that $H_{k}\left(X, X_{0} ; \mathbb{Z}\right)=\mathbb{Z}^{l}$.) Note that the matrices $\left(\alpha_{i j}^{k+1}\right)$ and $\left(\alpha_{i j}^{k+1}(1, \ldots, 1)\right)$ are presentation matrices of Coker $\partial_{k+1}$ and Coker $\partial_{k+1}^{\mathbb{Z}}$, respectively. Therefore

$$
\left|\Delta_{l}\left(H_{k}\left(X, X_{0} ; \Lambda_{\mu}\right)\right)(1, \ldots, 1)\right|=\left|\Delta_{l+r_{k}}\left(\operatorname{Coker} \partial_{k+1}\right)(1, \ldots, 1)\right|=1 .
$$

This completes the proof.

Proof of Theorem C (1) Let $W$ be a 1-solvable cobordism between $X_{L_{0}}$ and $X_{L_{1}}$. In particular, the inclusion induces $\mathbb{Z}^{\mu}=H_{1}\left(X_{L_{0}}\right) \cong H_{1}(W)$ and $H_{1}\left(W, X_{L_{0}}\right)=$ $H_{1}\left(W, X_{L_{1}}\right)=0$. By Poincaré duality and the universal coefficient theorem,

$$
H_{2}\left(W, X_{L_{0}}\right) \cong H^{2}\left(W, X_{L_{1}}\right) \cong \operatorname{Hom}_{\mathbb{Z}}\left(H_{2}\left(W, X_{L_{1}}\right), \mathbb{Z}\right)=\mathbb{Z}^{2 r} .
$$


(Since $W$ is a 1 -solvable cobordism between $X_{L_{0}}$ and $X_{L_{1}}, \operatorname{rank}_{\mathbb{Z}} H_{2}\left(W, X_{L_{1}}\right)$ is even.) Let $C_{*}=C_{*}\left(W, X_{L_{0}} ; \Lambda_{\mu}\right)$. Then

$$
H_{i}\left(C_{*} \otimes_{\Lambda_{\mu}} \mathbb{Z}\right)=H_{i}\left(W, X_{L_{0}} ; \mathbb{Z}\right)=0 \quad \text { for } i=0,1 .
$$

Since $\Lambda_{\mu}=\mathbb{Z}\left[\mathbb{Z}^{\mu}\right]$ and $\mathbb{Z}^{\mu}$ is a poly-torsion-free-abelian group, then by [15, Proposition 2.10],

$$
H_{i}\left(C_{*} \otimes_{\Lambda_{\mu}} \mathcal{K}\right)=H_{i}\left(W, X_{L_{0}} ; \Lambda_{\mu}\right) \otimes_{\Lambda_{\mu}} \mathcal{K}=0 \quad \text { for } i=0,1 .
$$

Similarly, $H_{i}\left(W, X_{L_{1}} ; \Lambda_{\mu}\right) \otimes_{\Lambda_{\mu}} \mathcal{K}=0$ for $i=0,1$. From duality and the universal coefficient theorem, $H_{i}\left(W, X_{L_{0}} ; \Lambda_{\mu}\right) \otimes_{\Lambda_{\mu}} \mathcal{K}=0$ for $i=3$, 4. So

$\operatorname{rank}_{\Lambda_{\mu}} H_{2}\left(W, X_{L_{i}} ; \Lambda_{\mu}\right)=\chi\left(C_{*}\right)=\chi\left(C_{*}\left(W, X_{L_{i}} ; \mathbb{Z}\right)\right)=\operatorname{rank}_{\mathbb{Z}} H_{2}\left(W, X_{L_{i}} ; \mathbb{Z}\right)=2 r$ for $i=0,1$. As in Lemma 5.2, the existence of a 1-Lagrangian and 1-duals implies that the following is exact for $i=0,1$ :

$$
t H_{2}\left(W, X_{L_{i}} ; \Lambda_{\mu}\right) \rightarrow H_{1}\left(X_{L_{i}} ; \Lambda_{\mu}\right) \rightarrow H_{1}\left(W ; \Lambda_{\mu}\right) \rightarrow t H_{1}\left(W, X_{L_{i}} ; \Lambda_{\mu}\right) .
$$

(Note that $H_{1}\left(W, X_{L_{i}} ; \Lambda_{\mu}\right)=t H_{1}\left(W, X_{L_{i}} ; \Lambda_{\mu}\right)$ for $i=0$, 1.) In particular, (1) is proved because

$$
\operatorname{rank}_{\Lambda_{\mu}} H_{1}\left(X_{L_{0}} ; \Lambda_{\mu}\right)=\operatorname{rank}_{\Lambda_{\mu}} H_{1}\left(W ; \Lambda_{\mu}\right)=\operatorname{rank}_{\Lambda_{\mu}} H_{1}\left(X_{L_{1}} ; \Lambda_{\mu}\right) .
$$

(2) For $i=0,1$, applying Lemma 5.1 to the exact sequence

$$
t H_{2}\left(W, X_{L_{i}} ; \Lambda_{\mu}\right) \rightarrow H_{1}\left(X_{L_{i}} ; \Lambda_{\mu}\right) \rightarrow H_{1}\left(W ; \Lambda_{\mu}\right) \rightarrow t H_{1}\left(W, X_{L_{i}} ; \Lambda_{\mu}\right),
$$

the following is also exact:

$$
t H_{2}\left(W, X_{L_{i}} ; \Lambda_{\mu}\right) \rightarrow t H_{1}\left(X_{L_{i}} ; \Lambda_{\mu}\right) \rightarrow t H_{1}\left(W ; \Lambda_{\mu}\right) \rightarrow t H_{1}\left(W, X_{L_{i}} ; \Lambda_{\mu}\right) .
$$

Denote the $0^{\text {th }}$ Alexander polynomial of these modules and $t H_{1}\left(\partial W ; \Lambda_{\mu}\right)$ by

$$
\Delta_{2, i}, \quad \Delta_{L_{i}}^{T}, \quad \Delta_{W}, \quad \Delta_{1, i} \quad \text { and } \quad \Delta_{\partial W}
$$

respectively.

In (1), we proved $\operatorname{rank}_{\Lambda_{\mu}} H_{1}\left(W, X_{L_{i}} ; \Lambda_{\mu}\right)=0$ using the fact that $H_{1}\left(W, X_{L_{i}}\right)=0$. Using an Euler characteristic argument, we also proved $\operatorname{rank}_{\Lambda_{\mu}} H_{2}\left(W, X_{L_{i}} ; \Lambda_{\mu}\right)=2 r$ where $H_{2}\left(W, X_{L_{i}}\right)=\mathbb{Z}^{2 r}$. Applying Lemma 5.4 to $\left(X, X_{0}\right)=\left(W, X_{L_{i}}\right)$ for the cases $(k, l)=(2,2 r)$ and $(1,0)$, we obtain $\left|\Delta_{2, i}(1, \ldots, 1)\right|=\left|\Delta_{1, i}(1, \ldots, 1)\right|=1$. Using Remark 5.3(3), there exist $g_{i}$ and $g_{i}^{\prime}$ in $\Lambda_{\mu}$ which divide $\Delta_{2, i}$ and $\Delta_{1, i}$, respectively and they satisfy $\Delta_{L_{i}}^{T} g_{i}=\Delta_{W} g_{i}^{\prime}$. Since $\left|g_{i}(1, \ldots, 1)\right|$ and $\left|g_{i}^{\prime}(1, \ldots, 1)\right|$ are nonnegative integers which divide 1 , they are 1 . Let $g=g_{0} g_{1}^{\prime}$ and $g^{\prime}=g_{0}^{\prime} g_{1}$. Then, $\Delta_{L_{0}}^{T} g \doteq \Delta_{W} g_{0}^{\prime} g_{1}^{\prime} \doteq \Delta_{L_{1}}^{T} g^{\prime}$ with $|g(1, \ldots, 1)|=\left|g^{\prime}(1, \ldots, 1)\right|=1$. 
Since $\Lambda_{\mu}$ is a unique factorization domain, we can split $\Delta_{L_{i}}^{T}=u_{i} v_{i}$ and $\Delta_{\partial W}=u v$ uniquely (up to units of $\Lambda_{\mu}$ ) so that $v_{0}, v_{1}, v$ consist of all irreducible factors $f \in \Lambda_{\mu}$ with $|f(1, \ldots, 1)| \neq 1$ in $\Delta_{L_{0}}^{T}, \Delta_{L_{1}}^{T}, \Delta_{\partial W}$. Now, $\Delta_{L_{0}}^{T} g \doteq \Delta_{L_{1}}^{T} g^{\prime}$ becomes $u_{0} v_{0} g \stackrel{\varphi}{=}$ $u_{1} v_{1} g^{\prime}$. Since $g, g^{\prime}, u_{0}$ and $u_{1}$ are factors with $|f(1, \ldots, 1)|=1, v_{0} \doteq v_{1}$.

From the Mayer-Vietoris sequence,

$H_{1}\left(\partial X_{L_{0}} ; \Lambda_{\mu}\right) \rightarrow H_{1}\left(X_{L_{0}} ; \Lambda_{\mu}\right) \oplus H_{1}\left(X_{L_{1}} ; \Lambda_{\mu}\right) \rightarrow H_{1}\left(\partial W ; \Lambda_{\mu}\right) \rightarrow H_{0}\left(\partial X_{L_{0}} ; \Lambda_{\mu}\right)$

is exact. Since the extremal terms are $\prod_{i=1}^{\mu}\left(t_{i}-1\right)$-torsion, we can apply Lemma 5.1 to get the following exact sequence:

$$
\begin{aligned}
t H_{1}\left(\partial X_{L_{0}} ; \Lambda_{\mu}\right) \rightarrow t H_{1}\left(X_{L_{0}} ; \Lambda_{\mu}\right) \oplus t H_{1}\left(X_{L_{1}} ; \Lambda_{\mu}\right) \rightarrow t H_{1}(\partial W & \left.; \Lambda_{\mu}\right) \\
& \rightarrow t H_{0}\left(\partial X_{L_{0}} ; \Lambda_{\mu}\right) .
\end{aligned}
$$

By Remark 5.3(3), $\Delta_{\partial W} \lambda \doteq \Delta_{L_{0}}^{T} \Delta_{L_{1}}^{T} \lambda^{\prime}$ for some factors $\lambda, \lambda^{\prime}$ of $\prod_{i=1}^{\mu}\left(t_{i}-1\right)$. By the reciprocity of Blanchfield pairing [1],

$$
\Delta_{L_{i}}^{T} \doteq \overline{\Delta_{L_{i}}^{T}} \quad \text { for } i=0,1 .
$$

In particular, $u_{0}=\bar{u}_{0}$. Now, we have

$$
u \doteq u_{0} u_{1} \doteq \bar{u}_{0} u_{1} .
$$

Recall that a linking pairing $(M, b)$ is neutral if $M$ has a submodule $N$ such that $N=N^{\perp}$. By Theorem B, we proved that the Blanchfield form of $\partial W$ is neutral. This implies that $\Delta_{\partial W}=h \bar{h}$ for some $h \in \Lambda_{\mu}$ by [28, Lemma 3.26]. In particular,

$$
u \doteq f \bar{f} \quad \text { for some } f \in \Lambda_{\mu} \text { with }|f(1, \ldots, 1)|=1 .
$$

Combining all these observations,

$$
\Delta_{L_{0}}^{T} f \bar{f} \doteq u_{0} v_{0} u=u_{0} \bar{u}_{0} u_{1} v_{0} \doteq u_{0} \bar{u}_{0} u_{1} v_{1} \doteq \Delta_{L_{1}}^{T} u_{0} \bar{u}_{0} .
$$

Here, $f$ and $u_{0}$ satisfy the conditions $|f(1, \ldots, 1)|=1,\left|u_{0}(1, \ldots, 1)\right|=1$. This completes the proof.

Remark It should be noted that Theorem $\mathrm{C}$ is not a direct consequence of Theorem B. From Theorem B without Lemma 5.4, one may deduce that if $X_{L_{0}}$ and $X_{L_{1}}$ are 1-solvable cobordant, then

(1) $\operatorname{rank}_{\Lambda_{\mu}} H_{1}\left(X_{L_{0}} ; \Lambda_{\mu}\right)=\operatorname{rank}_{\Lambda_{\mu}} H_{1}\left(X_{L_{1}} ; \Lambda_{\mu}\right)$,

(2) $\Delta_{L_{0}}^{T} f_{0} \overline{f_{0}} \doteq \Delta_{L_{1}}^{T} f_{1} \overline{f_{1}}$

for some $f_{0}, f_{1} \in \Lambda_{\mu S}-\{0\}$. Lemma 5.4 is crucial to obtain the stronger conclusion that we can choose $f_{0}, f_{1} \in \Lambda_{\mu}$ such that $\left|f_{0}(1, \ldots, 1)\right|=\left|f_{1}(1, \ldots, 1)\right|=1$. 
Finally, we mention what can be deduced from Theorems B and $\mathrm{C}$ for the special case of 2-component links with linking number 1 . Note that by the work of Levine [33], the Blanchfield form (without localization) $b_{L}: t H_{1}\left(X_{L} ; \Lambda_{2}\right) \times t H_{1}\left(X_{L} ; \Lambda_{2}\right) \rightarrow \mathcal{K} / \Lambda_{2}$ is nonsingular.

Corollary D Suppose $L$ is a 2-component link with linking number 1. If $X_{L}$ and $X_{H}$ are 1-solvable cobordant, then:

(1) $\left[b_{L}\right]=0 \in W\left(\mathcal{K}, \Lambda_{2},-\right)$.

(2) $\beta(L)=0$.

(3) $\quad \Delta_{0}(L) \doteq f \bar{f}$ for some $f \in \Lambda_{2}$ such that $|f(1,1)|=1$.

In particular, the conclusion holds if $L$ and $H$ are height-3 Whitney tower/grope concordant.

Proof Let $L$ be a 2-component link with linking number 1. Assume $X_{L}$ and $X_{H}$ are 1 -solvable cobordant. Since $X_{H}=S^{1} \times S^{1} \times I$ and the $\mathbb{Z} \oplus \mathbb{Z}$ cover of $X_{H}$ is $\mathbb{R} \times \mathbb{R} \times I,\left[b_{H}\right]=0, \beta(H)=0$ and $\Delta_{0}(H)=1$. This shows (1) and (2). With the notation in the proof of Theorem $\mathrm{C}$ (applied to $L_{0}=H$ and $L_{1}=L$ ), $u_{0}=1$ and

$$
\Delta_{0}(H) f \bar{f} \doteq \Delta_{0}(L) u_{0} \bar{u}_{0}
$$

for some $f \in \Lambda_{2}$ such that $|f(1,1)|=1$. Since $\Delta_{0}(H)=1$ and $u_{0}=1, \Delta_{0}(L)=f \bar{f}$ for some $f \in \Lambda_{2}$ such that $|f(1,1)|=1$. This completes the proof of (3).

\section{References}

[1] $\mathbf{R ~ C ~ B l a n c h f i e l d , ~ I n t e r s e c t i o n ~ t h e o r y ~ o f ~ m a n i f o l d s ~ w i t h ~ o p e r a t o r s ~ w i t h ~ a p p l i c a t i o n s ~ t o ~}$ knot theory, Ann. of Math. 65 (1957) 340-356 MR0085512

[2] J R Burke, Infection by string links and new structure in the knot concordance group, Algebr. Geom. Topol. 14 (2014) 1577-1626 MR3212578

[3] A J Casson, CM Gordon, On slice knots in dimension three, from: "Algebraic and geometric topology”, (R J Milgram, editor), Proc. Sympos. Pure Math. 32, Amer. Math. Soc. (1978) 39-53 MR520521

[4] A J Casson, C M Gordon, Cobordism of classical knots, from: "À la recherche de la topologie perdue", (L Guillou, A Marin, editors), Progr. Math. 62, Birkhäuser, Boston (1986) 181-199 MR900252

[5] J C Cha, Structure of the string link concordance group and Hirzebruch-type invariants, Indiana Univ. Math. J. 58 (2009) 891-927 MR2514393 
[6] J C Cha, Link concordance, homology cobordism and Hirzebruch-type defects from iterated p-covers, J. Eur. Math. Soc. (JEMS) 12 (2010) 555-610 MR2639313

[7] J C Cha, Amenable $L^{2}$-theoretic methods and knot concordance, Int. Math. Res. Not. 2014 (2014) 4768-4803 MR3257550

[8] J C Cha, Symmetric Whitney tower cobordism for bordered 3-manifolds and links, Trans. Amer. Math. Soc. 366 (2014) 3241-3273 MR3180746

[9] J C Cha, M Powell, Nonconcordant links with homology cobordant zero-framed surgery manifolds, Pacific J. Math. 272 (2014) 1-33 MR3270170

[10] D Cimasoni, A geometric construction of the Conway potential function, Comment. Math. Helv. 79 (2004) 124-146 MR2031702

[11] T D Cochran, S Harvey, C Leidy, Link concordance and generalized doubling operators, Algebr. Geom. Topol. 8 (2008) 1593-1646 MR2443256

[12] T D Cochran, S Harvey, C Leidy, Knot concordance and higher-order Blanchfield duality, Geom. Topol. 13 (2009) 1419-1482 MR2496049

[13] T D Cochran, S Harvey, C Leidy, Primary decomposition and the fractal nature of knot concordance, Math. Ann. 351 (2011) 443-508 MR2836668

[14] T D Cochran, T Kim, Higher-order Alexander invariants and filtrations of the knot concordance group, Trans. Amer. Math. Soc. 360 (2008) 1407-1441 MR2357701

[15] T D Cochran, K E Orr, P Teichner, Knot concordance, Whitney towers and $L^{2}-$ signatures, Ann. of Math. 157 (2003) 433-519 MR1973052

[16] T D Cochran, K E Orr, P Teichner, Structure in the classical knot concordance group, Comment. Math. Helv. 79 (2004) 105-123 MR2031301

[17] T D Cochran, P Teichner, Knot concordance and von Neumann $\rho$-invariants, Duke Math. J. 137 (2007) 337-379 MR2309149

[18] J Conant, R Schneiderman, P Teichner, Higher-order intersections in low-dimensional topology, Proc. Natl. Acad. Sci. USA 108 (2011) 8131-8138 MR2806650

[19] D Cooper, The universal abelian cover of a link, from: "Low-dimensional topology", (R Brown, T L Thickstun, editors), London Math. Soc. Lecture Note Ser. 48, Cambridge Univ. Press (1982) 51-66 MR662427

[20] R H Crowell, R H Fox, Introduction to knot theory, Graduate Texts in Math. 57, Springer, New York (1977) MR0445489

[21] C W Davis, Von Neumann rho-invariants and torsion in the topological knot concordance group, Algebr. Geom. Topol. 12 (2012) 753-789 MR2914617

[22] B D Franklin, The effect of infecting curves on knot concordance, Int. Math. Res. Not. 2013 (2013) 184-217 MR3041699

[23] M H Freedman, F Quinn, Topology of 4-manifolds, Princeton Math. Series 39, Princeton Univ. Press (1990) MR1201584 
[24] S Friedl, M Powell, An injectivity theorem for Casson-Gordon type representations relating to the concordance of knots and links, Bull. Korean Math. Soc. 49 (2012) 395-409 MR2934490

[25] S Friedl, M Powell, Links not concordant to the Hopf link, Math. Proc. Cambridge Philos. Soc. 156 (2014) 425-459 MR3181634

[26] S L Harvey, Homology cobordism invariants and the Cochran-Orr-Teichner filtration of the link concordance group, Geom. Topol. 12 (2008) 387-430 MR2390349

[27] A Hatcher, Algebraic topology, Cambridge Univ. Press (2002) MR1867354

[28] J Hillman, Algebraic invariants of links, 2nd edition, Series on knots and everything 52, World Scientific Publishing Co. Pte. Ltd. (2012) MR2931688

[29] P D Horn, The nontriviality of the grope filtrations of the knot and link concordance groups, Comment. Math. Helv. 85 (2010) 751-773 MR2718138

[30] A Kawauchi, On the Alexander polynomials of cobordant links, Osaka J. Math. 15 (1978) 151-159 MR0488022

[31] C Kearton, Cobordism of knots and Blanchfield duality, J. London Math. Soc. 10 (1975) 406-408 MR0385873

[32] J P Levine, Knot cobordism groups in codimension two, Comment. Math. Helv. 44 (1969) 229-244 MR0246314

[33] J P Levine, The module of a 2-component link, Comment. Math. Helv. 57 (1982) 377-399 MR689070

[34] J P Levine, Link invariants via the eta-invariant, Comment. Math. Helv. 69 (1994) 82-119 MR1259607

[35] R Schneiderman, Whitney towers and gropes in 4-manifolds, Trans. Amer. Math. Soc. 358 (2006) 4251-4278 MR2231378

Department of Mathematics, Pohang University of Science and Technology

Gyungbuk 790-784, South Korea

kminhoon@gmail.com

Received: 8 July $2014 \quad$ Revised: 20 October 2014 
